\title{
A generalization of Liu-Yau's quasi-local mass
}

\author{
Mu-Tao Wang and Shing-Tung Yau
}

\begin{abstract}
In $[11,12]$, Liu and the second author propose a definition of the quasi-local mass and prove its positivity. This is demonstrated through an inequality which in turn can be interpreted as a total mean curvature comparison theorem for isometric embeddings of a surface of positive Gaussian curvature. The Riemannian version corresponds to an earlier theorem of Shi and Tam [18]. In this article, we generalize such an inequality to the case when the Gaussian curvature of the surface is allowed to be negative. This is done by an isometric embedding into the hyperboloid in the Minkowski space and a future-directed time-like quasi-local energy-momentum is obtained.
\end{abstract}

\section{Introduction}

Let $\left(\Omega, g_{i j}, p_{i j}\right)$ be a compact spacelike hypersurface in a time orientable four-dimensional spacetime $N$, where $g_{i j}$ is the induced metric and $p_{i j}$ is the second fundamental form of $\Omega$ in $N$. We assume the dominant energy condition holds on $\Omega$, i.e.,

$$
\mu \geq\left(\sum_{i} J^{j} J_{i}\right)^{1 / 2}
$$

where

$$
\mu=\frac{1}{2}\left[R-\sum_{i, j} p^{i j} p_{i j}+\left(\sum_{i} p_{i}^{i}\right)^{2}\right]
$$

and

$$
J^{i}=\sum_{j} D_{j}\left[p^{i j}-\left(\sum_{k} p_{k}^{k}\right) g^{i j}\right]
$$

and $R$ is the scalar curvature of the metric $g_{i j}$. Such a three-manifold $\left(\Omega, g_{i j}, p_{i j}\right)$ is called an initial data set. 
Liu and Yau prove the following theorem in $[11,12]$ :

Theorem 1.1. Let $\left(\Omega, g_{i j}, p_{i j}\right)$ be a compact initial data set. Suppose the boundary of $\Omega$ is a smooth surface $\Sigma$ with Gaussian curvature $K$ and mean curvature $H$ with respect to the outward normal. If $K>0$ and $H>\left|\operatorname{tr}_{\Sigma} p\right|$, then

$$
\int_{\Sigma} H_{0}-\int_{\Sigma} \sqrt{H^{2}-\left(\operatorname{tr}_{\Sigma} p\right)^{2}} \geq 0
$$

where $H_{0}$ is the mean curvature of the (essentially unique) isometric embedding $F_{0}$ of $\Sigma$ into $\mathbb{R}^{3}$. The equality holds only if $N$ is a flat spacetime along $\Omega$.

We remark that $\sqrt{H^{2}-\left(\operatorname{tr}_{\Sigma} p\right)^{2}}$ is the Lorentz norm of the mean curvature vector of $\Sigma$ in $N$. Liu and Yau (see also Kijowski [10]) propose to define the quasi-local mass of $\Sigma$ to be $\int_{\Sigma} H_{0}-\int_{\Sigma} \sqrt{H^{2}-\left(\operatorname{tr}_{\Sigma} p\right)^{2}}$. The inequality amounts to the positivity of Liu-Yau's quasi-local mass. Liu-Yau's theorem generalizes the Riemannian version of this inequality which was proved earlier by Shi and Tam [18]:

Theorem 1.2. Let $\Omega$ be a compact three-manifold with positive scalar curvature. Suppose the boundary of $\Omega$ is a smooth surface $\Sigma$ with positive Gausssian curvature and positive mean curvature $H$ with respect to the outward normal. Then

$$
\int_{\Sigma}\left(H_{0}-H\right) \geq 0
$$

where $H_{0}$ is the mean curvature of the (essentially unique) isometric embedding $F_{0}$ of $\Sigma$ into $\mathbb{R}^{3}$. The inequality holds only if $\Omega$ is flat.

The expression $\int_{\Sigma}\left(H_{0}-H\right)$ is indeed the quasi-local mass of Brown and York $[4,5]$. Liu-Yau's theorem for time symmetric space time $\left(p_{i j}=0\right.$ on $\Omega$ ) implies the Riemmanian version. Indeed, the validity of Liu-Yau's theorem relies only on the fact that $\Sigma$ bounds a space-like three-manifold $\Omega$, but not on any particular $\Omega$ (the expression $\int_{\Sigma} \sqrt{H^{2}-\left(\operatorname{tr}_{\Sigma} p\right)^{2}}$ is independent of $\Omega$ ).

In this article, we generalize Liu-Yau's quasi-local mass in the case when the Gaussian curvature of the surface is not necessarily positive. In addition, we obtain a time-like four-vector instead of a positive quantity. The motivation for such a generalization is the following. First of all, in general relativity, it is desirable to extend the definition of quasi-local mass to non-convex surfaces in order to deal with, for example, black hole collision. Secondly, 
we intend to resolve the issue of momentum in Liu-Yau's definition. It was pointed out in $[15]$ that there exists surfaces in $\mathbb{R}^{3,1}$ with strictly positive Liu-Yau quasi-local mass. In Liu-Yau's formulation, the mass is zero only if $\Sigma$ lies in a totally geodesic $\mathbb{R}^{3}$. We believe the momentum has to be accounted for. We compare to isometric embedding of $\Sigma$ into $\mathbb{R}^{3,1}$ and a four-vector naturally arises in such a setting.

We prove the following theorem.

Theorem 1.3. Let $\left(\Omega, g_{i j}, p_{i j}\right)$ be a compact initial data set. Suppose the boundary of $\Omega$ is a smooth surface $\Sigma$ homeomorphic to the two-sphere. Let $K$ be the Gaussian curvature and $H$ be the mean curvature with respect to the outward normal of $\Sigma$. Suppose $\kappa>0$ satisfies $K>-\kappa^{2}$ and $H>\left|\operatorname{tr}_{\Sigma} p\right|$. Let $F_{0}$ be the (essentially unique) isometric embedding of $\Sigma$ into $\mathbb{H}_{-\kappa^{2}}^{3} \subset \mathbb{R}^{3,1}$. Then on $\Sigma$ there exists a future-directed time-like vector-valued function $\mathbf{W}^{0}: \Sigma \rightarrow \mathbb{R}^{3,1}$ which depends only on $\sqrt{H^{2}-\left(\operatorname{tr}_{\Sigma} p\right)^{2}}$ and the embedding of $\Sigma$ into $\mathbb{H}_{-\kappa^{2}}^{3}$ such that

$$
\int_{\Sigma}\left[H_{0}-\sqrt{H^{2}-\left(\operatorname{tr}_{\Sigma} p\right)^{2}}\right] \mathbf{W}^{0}
$$

is a future-directed non-space-like vector. Here, $H_{0}$ is the mean curvature of the isometric embedding into $\mathbb{H}_{-\kappa^{2}}^{3}$.

Our theorem is in the spirit of Liu-Yau's as the expression depends only on the metric and the embedding of $\Sigma$ and $\sqrt{H^{2}-\left(\operatorname{tr}_{\Sigma} p\right)^{2}}$ and is thus independent of the particular $\Omega$. Theorem 1.3 is not sharp even if $\Omega$ itself is part of a hyperbolic space. But it does recover Liu-Yau's theorem as $\kappa \rightarrow 0$.

The Riemannian version is

Theorem 1.4. Let $\Omega$ be a compact three-manifold with scalar curvature $R \geq-6 \kappa^{2}$, for some $\kappa>0$. Suppose the boundary of $\Omega$ is a smooth surface $\Sigma$ homeomorphic to the two-sphere. We assume $\Sigma$ has positive mean curvature $H$ with respect to the outward normal and Gaussian curvature $K>-\kappa^{2}$. Then there exists a future-directed time-like vector-valued function $\mathbf{W}^{0}: \Sigma \rightarrow \mathbb{R}^{3,1}$ which depends on $H$ and the embedding of $\Sigma$ into $\mathbb{H}_{-\kappa^{2}}^{3}$ such that

$$
\int_{\Sigma}\left(H_{0}-H\right) \mathbf{W}^{0}
$$

is a future-directed non-space-like vector. Here, $H_{0}$ is the mean curvature of the isometric embedding into $\mathbb{H}_{-\kappa^{2}}^{3}$. 
$\mathbf{W}^{0}$ comes from the solution of the backward parabolic equation (5.3) and is related to the square norm of the Killing spinor on $\mathbb{H}_{-\kappa^{2}}^{3} \cdot \mathbf{W}^{0}$ approaches a constant vector as $\kappa \rightarrow 0$. The comparison theorem holds when $\Omega$ has more than one component and in higher dimension.

A common feature of Shi-Tam's and Liu-Yau's theorem is an idea of Bartnik [2] (see also [23]) which is to glue together $\Omega$ with the outer component of $\mathbb{R}^{3} \backslash F_{0}(\Sigma)$ along $\Sigma$. Pushing $F_{0}(\Sigma)$ along the outward normal direction gives a natural foliation of the outer component. In Shi-Tam's case, the joint is smoothed out by perturbing the flat metric in the transverse direction of the foliation so that the mean curvatures at the joint agree and the new metric has zero scalar curvature and is asymptotically flat. The proof is followed by the monotonicity formula of a mass expression and the positive mass theorem for such a manifold. Liu and Yau were able to deal with the general space-time case. The key point was a procedure followed in the proof of the positive mass theorem by Schoen and Yau [21]. Out of an initial data set $\left(\Omega, g_{i j}, p_{i j}\right)$, they constructed a new three-manifold with zero scalar curvature while the original information of $p_{i j}$ was retained. The mean curvature is no longer continuous along the joint. Nevertheless, through a delicate estimate, they were able to prove the existence of harmonic spinors to furnish the proof of the positivity of the total mass. Positive mass theorems on manifolds with discontinuities have been proved by Shi-Tam [18] and Miao [13].

In our case, we suppose $\Sigma$ has Gaussian curvature $K>-\kappa^{2}$, for some $\kappa>0$. By a theorem of Pogorelov [17], $\Sigma$ can be isometrically embedded into the hyperbolic space $\mathbb{H}_{-\kappa^{2}}^{3}$ of constant sectional curvature $-\kappa^{2}$, and the embedding is unique up to a hyperbolic isometry in $\mathrm{SO}(3,1) . \mathbb{H}_{-\kappa^{2}}^{3}$ is identified with the hyperboloid in the Minkowski space $\mathbb{R}^{3,1}$; so this becomes an embedding of $\Sigma$ into $\mathbb{R}^{3,1}$. Such embeddings are unique only when restricted to $\mathbb{H}_{-\kappa^{2}}^{3}$.

We remark that the second fundamental form of $F_{0}$ is positive definite by the Gauss formula. In particular, the mean curvature $H_{0}>0$. Indeed the Gauss formula says the sectional curvature $K_{a b}$ satisfies

$$
K_{a b}=-\kappa^{2}+h_{a a} h_{b b}-h_{a b}^{2}
$$

where $h_{a b}$ is the second fundamental form.

Our proof involves a construction similar to that of Bartnik, Shi-Tam, and Liu-Yau. We glue $\Omega$ with the outer component of $\mathbb{H}_{-\kappa^{2}}^{3} \backslash F_{0}(\Sigma)$ by identifying the two embeddings and perturbing the hyperbolic metric in the transverse direction so that the scalar curvature remains $-6 \kappa^{2}$ and the metric is asymptotically hyperbolic. We also introduce the function $\mathbf{W}$ by 
solving a backward parabolic equation with a prescribed value at infinity. We show that the difference of the weighted total mean curvature of the leaves in the two metrics is monotone and is positive at infinity by a positive mass theorem for asymptotically hyperbolic manifolds.

We remark that the positive mass theorems for the ADM mass (asymptotically flat) and the Bondi mass were first proved by Schoen and Yau [19-22]. Witten [25] (see also [16]) then gave a different yet simpler proof using a spinor argument. This argument is adapted by several authors $[14,1,24,7,8,26]$ to study the mass and rigidity of asymptotically hyperbolic manifolds. The formulation of the positive mass theorem for asymptotically hyperbolic manifolds is more complicated than the asymptotically flat case in that the non-trivial Killing spinor is involved. Our definition of mass involves a particular foliation asymptotic to surfaces of constant mean curvature. A perhaps more canonical one is the foliation by surfaces of constant mean curvature constructed by Huisken and Yau [9]. We plan to investigate this direction in the near future.

The paper is organized as the follows: In Section 2, we study the foliation of the hyperbolic space and derive the growth estimates of the relevant geometric quantities. Through the prescribed scalar curvature equation, we obtain an asymptotically hyperbolic three-manifold $\left(M, g^{\prime \prime}\right)$ with scalar curvature $-6 \kappa^{2} . M$ is diffeomorphic to $\mathbb{H}_{-\kappa^{2}}^{3} \backslash \Omega_{0}$, where $\Omega_{0}$ is the region in $\mathbb{H}_{-\kappa^{2}}^{3}$ enclosed by $F_{0}(\Sigma)$. The mean curvature of the inner boundary of $M$ can be prescribed to be any positive function $\mathcal{H}$. In Section 3, we review Witten's Lichnerowicz formula for the hypersurface spin connection, and we express the total mass of the $\left(M, g^{\prime \prime}\right)$ as the limit of an integral on the leave of the foliation. In Section 4, we study the Killing spinors on $\mathbb{H}_{-\kappa^{2}}^{3}$ and calculate the total mass of $\left(M, g^{\prime \prime}\right)$ explicitly. In Section 5 , we derive the monotonicity formula of the mass expression. In Section 6 , we prove the positivity of the total mass of $\left(M, g^{\prime \prime}\right)$ by gluing with $\Omega$ and choosing a suitable $\mathcal{H}$. The proofs of Theorem 1.3 and 1.4 are given at the end of Section 6 .

\section{Foliations with prescribed scalar curvature}

\subsection{Foliations on hyperbolic spaces}

Let $\Sigma$ be any $(n-1)$-dimensional Riemannian manifold. We assume each sectional curvature of $\Sigma$ is not less than $-\kappa^{2}$. Let $F_{0}: \Sigma \rightarrow \mathbb{H}_{-\kappa^{2}}^{n}$ be an isometric embedding and denote the image by $\Sigma_{0}=F_{0}(\Sigma)$. We deform $\Sigma_{0}$ in the normal direction at unit speed in order to obtain a foliation of the outer region of the surface $\Sigma_{0}$. This can be described by an ODE: for each 
$p \in \Sigma$, we consider

$$
\begin{cases}\frac{d}{d r} F(p, r) & =N(p, r) \\ F(p, 0) & =F_{0}(p)\end{cases}
$$

where $F: \Sigma \times[0, \infty) \rightarrow \mathbb{H}_{-\kappa^{2}}^{n}$ and $N$ is the unit outward normal of the surface $\Sigma_{r}=F(\Sigma, r)$. The parameter $r$ represents the distance function to $\Sigma_{0}$, and $\Sigma_{r}$ are exactly the level sets of $r$. For each fixed $p \in \Sigma, F(p, r), 0 \leq r<$ $\infty$, is a unit speed geodesic.

We fix a coordinate system $\left(x^{1}, \ldots, x^{n-1}\right)$ on $\Sigma$, and this gives a parametrization of each leaf $\Sigma_{r}$. Let $g_{a b}(p, r)=\left\langle\frac{\partial F}{\partial x^{a}}, \frac{\partial F}{\partial x^{b}}\right\rangle, a, b=1 \ldots n-1$, be the induced metric on the leave $\Sigma_{r}$. Therefore, the hyperbolic metric can be written as $d r^{2}+g_{a b}(p, r)$. For each $p, g_{a b}(p, r)$ satisfies the ODE

$$
\frac{d}{d r} g_{a b}(p, r)=2 h_{a b}(p, r)
$$

where $h_{a b}(p, r)=\left\langle\nabla_{\frac{\partial F}{\partial x^{a}}} N, \frac{\partial F}{\partial x^{b}}\right\rangle$ is the second fundamental form of $\Sigma_{r}$. By the assumption of sectional curvature, $h_{a b}(p, 0)>0$ for each $p \in \Sigma$. $h_{a b}$ satisfies

$$
\frac{d}{d r} h_{a b}=g^{c d} h_{a c} h_{b d}-R\left(\frac{\partial F}{\partial x^{a}}, N, \frac{\partial F}{\partial x^{b}}, N\right)
$$

In our case, $R\left(\frac{\partial F}{\partial x^{a}}, N, \frac{\partial F}{\partial x^{b}}, N\right)=-\kappa^{2} g_{a b}$ and $h_{b}^{a}=g^{a c} h_{c b}$ satisfies

$$
\frac{d}{d r} h_{b}^{a}=-h_{c}^{a} h_{b}^{c}+\kappa^{2} \delta_{b}^{a}
$$

The mean curvature $H_{0}=g^{a b} h_{a b}$ satisfies

$$
\frac{d}{d r} H_{0}=-|A|^{2}+(n-1) \kappa^{2}
$$

Equation (2.3) is an integrable first order ODE system. Given any point $p \in \Sigma$, choose a coordinate system so that $h_{a b}(p, 0)=\lambda_{a}(p, 0) g_{a b}(p, 0)$ and $h_{b}^{a}(p, 0)=\lambda_{a}(p, 0) \delta_{b}^{a}$ is diagonalized with principal curvature $\lambda_{a}(p, 0)$. By the uniqueness of ODE system, the solution is a diagonal matrix $h_{b}^{a}(p, r)=$ 
$\lambda_{a}(p, r) \delta_{b}^{a}$ and the principal curvatures $\lambda_{a}=\lambda_{a}(p, r)$ satisfy

$$
\frac{d}{d r} \lambda_{a}=-\lambda_{a}^{2}+\kappa^{2}
$$

It is easy to see in case when $\lambda_{a}(p, 0) \geq \kappa, \lambda_{a}(p, r)=\kappa \operatorname{coth}\left(\kappa\left(\mu_{a}+r\right)\right)$ with $\lambda_{a}(p, 0)=\kappa \operatorname{coth}\left(\kappa \mu_{a}\right)$ is a solution of this ODE and $\lim _{r \rightarrow \infty} \lambda_{a}=\kappa$ is independent of the initial condition. If $\lambda_{a}(p, 0)<\kappa$, we may replace coth by tanh and the results are similar. Since only the asymptotic behavior at infinity will be relevant, for simplicity we shall restrict to the case $\lambda_{a}(p, 0) \geq$ $\kappa$ in the following discussions.

Now we can solve Equation (2.1). Since $\lambda_{a}=\kappa \operatorname{coth}\left(\kappa\left(\mu_{a}+r\right)\right)$ are the eigenvalues of $h_{b}^{a}$, we have $h_{a b}=\kappa \operatorname{coth}\left(\kappa\left(\mu_{a}+r\right)\right) g_{a b}$, and the $g_{a b}$ satisfy

$$
\frac{d}{d r} g_{a b}=2 \kappa \operatorname{coth}\left(\kappa\left(\mu_{a}+r\right)\right) g_{a b}
$$

We may assume $g_{a b}(p, 0)=\delta_{a b}$ by choosing coordinates near $p$. Since the solution of initial value problem

$$
\left\{\begin{array}{l}
\frac{d}{d r} \eta_{a}=2 \kappa \operatorname{coth}\left(\kappa\left(\mu_{a}+r\right)\right) \eta_{a} \\
\eta_{a}(0)=1
\end{array}\right.
$$

is $\eta_{a}(r)=\frac{\sinh ^{2}\left(\kappa\left(\mu_{a}+r\right)\right)}{\sinh ^{2}\left(\kappa \mu_{a}\right)}$, we obtain

$$
g_{a b}(p, r)=\frac{\sinh ^{2}\left(\kappa\left(\mu_{a}+r\right)\right)}{\sinh ^{2}\left(\kappa \mu_{a}\right)} \delta_{a b} .
$$

The volume element of $\Sigma_{r}$ is thus

$$
\sqrt{\operatorname{det} g_{a b}(p, r)}=\prod_{a=1}^{n-1} \frac{\sinh \left(\kappa\left(\mu_{a}+r\right)\right)}{\sinh \left(\kappa \mu_{a}\right)} \sqrt{\operatorname{det} g_{a b}(p, 0)} .
$$

It is clear that $\tilde{g}_{a b}(p, r)=e^{-2 \kappa r} g_{a b}(p, r)$ is uniformly equivalent to the standard metric of $\mathbb{S}^{n-1}$ for any $r$.

The mean curvature of $\Sigma_{r}$ is

$$
H_{0}(p, r)=\sum_{a=1}^{n-1} \kappa \operatorname{coth}\left(\kappa\left(\mu_{a}+r\right)\right)
$$


By the Gauss formula, the sectional curvature $K_{a b}$ is

$$
K_{a b}(p, r)=-\kappa^{2}+\kappa^{2} \operatorname{coth}\left(\kappa\left(\mu_{a}+r\right)\right) \operatorname{coth}\left(\kappa\left(\mu_{b}+r\right)\right),
$$

and the scalar curvature $R^{r}$ of $\Sigma_{r}$ is

$$
R^{r}(p, r)=-(n-1)(n-2) \kappa^{2}+2 \kappa^{2} \sum_{a<b} \operatorname{coth}\left(\kappa\left(\mu_{a}+r\right)\right) \operatorname{coth}\left(\kappa\left(\mu_{b}+r\right)\right) .
$$

The limits are

$$
\lim _{r \rightarrow \infty} H_{0}(p, r)=(n-1) \kappa, \quad \lim _{r \rightarrow \infty} K_{a b}(p, r)=0 \quad \text { and } \lim _{r \rightarrow \infty} R^{r}(p, r)=0 .
$$

It is useful to view the total space as $\Sigma \times[0, \infty)$ with the metric $g_{a b}(r)$ on each $r$-slice. In the case when $n=3$, the normalized metric $\tilde{g}_{a b}=e^{-2 \kappa r} g_{a b}$ has scalar curvature $e^{2 \kappa r} R^{r}$ which approaches

$$
4 \kappa^{2} \frac{e^{\kappa\left(\mu_{1}-\mu_{2}\right)}+e^{\kappa\left(\mu_{2}-\mu_{1}\right)}}{e^{\kappa\left(\mu_{1}+\mu_{2}\right)}} .
$$

Unlike the flat case (see [18]), this is in general not a round metric on the sphere.

Another approach to deriving formulae in this section is to express the embedding of $\Sigma_{r}$ in terms of the coordinate function of $\mathbb{R}^{3,1}$ :

$$
\mathbf{X}(F(p, r))=\cosh \kappa r \mathbf{X}(F(p, 0))+\frac{\sinh \kappa r}{\kappa} \mathbf{N}(p, 0),
$$

where $\mathbf{N}(p, 0)$ is the outward normal of $\Sigma_{0}$ tangent to $\mathbb{H}_{-\kappa^{2}}^{3}$ as a vector in $\mathbb{R}^{3,1}$.

All the formulae in this section can be verified by this explicit embedding. Also, the normalization $e^{-\kappa r} \mathbf{X}(F(p, r))$ approaches $\mathbf{X}+\frac{1}{\kappa} \mathbf{N}$ which lies in the light cone.

\subsection{Prescribed scalar curvature equation}

Following the assumption in the previous section, we suppose $\Sigma_{0}$ bounds a region $\Omega_{0} \subset \mathbb{H}_{-\kappa^{2}}^{3}$, and denote $M=\mathbb{H}_{-\kappa^{2}}^{n} \backslash \Omega_{0}$. By the assumption on the sectional curvature of $\Sigma$, the hyperbolic metric $g^{\prime}$ on $M$ can be written as $d r^{2}+g_{a b}(p, r)$ where $r$ is the geodesic distance to $\Sigma_{0}$, and $g_{a b}(p, r)$ is the induced metric on the level set $\Sigma_{r}$ of $r$. The mean curvature of $\Sigma_{r}$ with respect to the outward normal in the hyperbolic metric is denoted 
by $H_{0}$. We consider a new metric $g^{\prime \prime}$ on $M$ of the form $u^{2} d r^{2}+g_{a b}(p, r)$ with prescribed scalar curvature $-n(n-1) \kappa^{2}$. Notice that $g^{\prime \prime}=u^{2} d r^{2}+$ $g_{a b}(p, r)$ induces the same metric on the leaf $\Sigma_{r} . u$ then satisfies the following parabolic PDE (see Equation (1.10) in [18]):

$$
2 H_{0} \frac{\partial u}{\partial r}=2 u^{2} \Delta_{r} u+\left(u-u^{3}\right)\left(R^{r}+n(n-1) \kappa^{2}\right),
$$

where $\Delta_{r}$ is the Laplace operator and $R^{r}$ is the scalar curvature of $\Sigma_{r}$. We also require the initial condition

$$
u(p, 0)=\frac{H_{0}(p, 0)}{\mathcal{H}(p)}
$$

to be satisfied where $\mathcal{H}$ is a positive function defined on $\Sigma$. The mean curvature of $\Sigma_{r}$ in the new metric is then $\mathcal{H}(p, r)=\frac{1}{u} H_{0}(p, r)$.

For simplicity, we shall focus on the $n=3$ case in the rest of the section. The general case can be derived similarly. The solution of

$$
2 H_{0} \frac{\partial u}{\partial r}=2 u^{2} \Delta_{r} u+\left(u-u^{3}\right)\left(R^{r}+6 \kappa^{2}\right)
$$

can be compared to the solution of the ODE

$$
\frac{d}{d r} f=h(r)\left(f-f^{3}\right), \quad f(0)=\min _{p \in \Sigma} u(p, 0),
$$

where $h(r)=\min _{x \in \Sigma_{r}} \frac{R^{r}+6 \kappa^{2}}{2 H_{0}}$. The solution of $(2.12)$ is

$f=\left(1+K \exp \left(-2 \int_{0}^{r} h(r) d r\right)\right)^{-1 / 2}$, where $K$ satisfies $f(0)=(1+K)^{-1 / 2}$.

By the maximum principle, we have $u(p, r) \geq \min \{f(r), 1\}>0$. The upper bound for $u$ can be obtained similarly. From these, it is not hard to see that $u$ satisfies the $C^{0}$ estimate:

$$
|u-1|<C e^{-3 \kappa r}
$$

We prove the following result.

Theorem 2.1. Let $\Sigma_{0}$ be an embedded convex surface in $\mathbb{H}_{-\kappa^{2}}^{3}$, and let $\Omega_{0}$ be the region enclosed by $\Sigma_{0}$ in $\mathbb{H}_{-\kappa^{2}}^{3}$. Let $M=\mathbb{H}_{-\kappa^{2}}^{3} \backslash \Omega_{0}$ and $\Sigma_{r}$ be the level set of the distance function $r$ to $\Sigma_{0}$. Let $g^{\prime}$ be the hyperbolic metric on $M$ 
which can be written as the form $g^{\prime}=d r^{2}+g_{a b}(p, r)$, where $g_{a b}(p, r)$ is the induced metric on $\Sigma_{r}$. Let $u$ be the solution of

$$
\left\{\begin{array}{l}
2 H_{0} \frac{\partial u}{\partial r}=2 u^{2} \Delta_{r} u+\left(u-u^{3}\right)\left(R^{r}+6 \kappa^{2}\right) \\
u(p, 0)=\frac{H_{0}(p, 0)}{\mathcal{H}(p)}
\end{array}\right.
$$

for a positive function $\mathcal{H}(p)$ defined on $\Sigma_{0}$. Here, $H_{0}$ is the mean curvature, $\Delta_{r}$ is the Laplace operator and $R^{r}$ is the scalar curvature of $\Sigma_{r}$. Then

(1) The solution exists for all time and

$$
\lim _{r \rightarrow \infty} e^{3 \kappa r}(u-1)=v_{\infty}
$$

is a smooth function.

(2) $g^{\prime \prime}=u^{2} d r^{2}+g_{a b}(p, r)$ is a complete asymptotically hyperbolic metric on $M$ with scalar curvature $-6 \kappa^{2}$.

Proof. We recall the expressions of the mean curvature $H_{0}$ and the scalar curvature $R^{r}$ of $\Sigma_{r}$ in the $n=3$ case:

$$
\begin{aligned}
H_{0} & =\kappa\left(\operatorname{coth}\left(\kappa\left(\mu_{1}+r\right)\right)+\operatorname{coth}\left(\kappa\left(\mu_{2}+r\right)\right)\right), \\
R^{r} & =2 \kappa^{2}\left(\operatorname{coth}\left(\kappa\left(\mu_{1}+r\right)\right) \operatorname{coth}\left(\kappa\left(\mu_{2}+r\right)\right)-1\right) .
\end{aligned}
$$

Denote $v=e^{3 \kappa r}(u-1)$. Then $v$ satisfies

$$
\frac{\partial}{\partial r} v=\frac{u^{2}}{H_{0}} \Delta_{r} v+\left[3 \kappa-\frac{u(u+1)\left(R^{r}+6 \kappa^{2}\right)}{2 H_{0}}\right] v .
$$

By (2.15), we have

$$
\frac{R^{r}+6 \kappa^{2}}{H_{0}}-3 \kappa=O\left(e^{-2 \kappa r}\right)
$$

and thus by $(2.13)$

$$
3 \kappa-\frac{u(u+1)\left(R^{r}+6 \kappa^{2}\right)}{2 H_{0}}=O\left(e^{-2 \kappa r}\right) .
$$

Define $\tilde{\Delta}_{r}=e^{2 \kappa r} \Delta_{r}$. Then $\tilde{\Delta}_{r}$ is uniformly equivalent to the Laplace operator of the standard metric on $S^{2}$. When $n-1=2$, this is the Laplace 
operator of $\tilde{g}_{a b}$ by the conformal invariance. Thus,

$$
2 e^{2 \kappa r} \frac{\partial}{\partial r} v=\frac{2 u^{2}}{H_{0}} \tilde{\Delta} v+O(1) v
$$

Take $t=-\frac{1}{4 \kappa} e^{-2 \kappa r}$. Then $2 e^{2 \kappa r} \frac{\partial}{\partial r}=\frac{\partial}{\partial t}$ and the equation becomes

$$
\frac{\partial}{\partial t} v=\frac{2 u^{2}}{H_{0}} \tilde{\Delta} v+O(1) v
$$

This equation holds for $t=-\frac{1}{4 \kappa}(r=0)$ to $t=0(r=\infty)$. It is not hard to show that the solution exists and converges to a smooth function $v_{\infty}$ on $\Sigma_{0}$, and we have

$$
\lim _{r \rightarrow \infty} e^{-3 \kappa r}(u-1)=v_{\infty}
$$

We can then apply the standard Schauder estimate to get derivative bounds for $u$.

We define the gauge transformation as in [1]:

$$
A:\left(T M, g^{\prime}\right) \longrightarrow\left(T M, g^{\prime \prime}\right)
$$

by $A\left(\frac{\partial}{\partial r}\right)=\frac{1}{u} \frac{\partial}{\partial r}$ and $A(X)=X$ for all $X \in T \Sigma_{r}$, or $A=\frac{1}{u} d u \otimes \frac{\partial}{\partial u}+e^{a} \otimes$ $e_{a}$. We can then check that $|A-I|=O\left(e^{-3 \kappa r}\right)$ and $\left|\nabla^{\prime} A\right|=O\left(e^{-3 \kappa r}\right)$.

\section{Lichnerowicz formula and the mass expression}

Let $\left(\Omega, g_{i j}\right)$ be a compact three-manifold with boundary $\partial \Omega$. Let $\left\{e_{i}\right\}_{i=1,2,3}$ be a local orthonormal frame on $\Omega$. We choose the frame so that $e_{3}$ is the outward normal to $\partial \Omega$ and $\left\{e_{a}\right\}_{a=1,2}$ are tangent to $\partial \Omega$.

Let $\nabla$ denote the Riemannian spin connection and $\nabla^{\partial \Omega}$ be the connection when restricted to $\partial \Omega$. Recall the Killing connection $\widehat{\nabla}$ is defined by

$$
\widehat{\nabla}_{V}=\nabla_{V}+\frac{\sqrt{-1}}{2} \kappa c(V) .
$$

The relations among these connections are

$$
\begin{aligned}
\widehat{\nabla}_{e_{a}} \psi & =\nabla_{e_{a}} \psi+\frac{\sqrt{-1}}{2} \kappa c\left(e_{a}\right) \cdot \psi \\
& =\nabla_{e_{a}}^{\partial \Omega} \psi+\frac{1}{2} \sum_{b=1}^{2} h_{a b} c\left(e_{3}\right) \cdot c\left(e_{b}\right) \cdot \psi+\frac{\sqrt{-1}}{2} \kappa c\left(e_{a}\right) \cdot \psi
\end{aligned}
$$


for $a=1,2$. Here, $h_{a b}=\left\langle\nabla_{e_{a}} e_{3}, e_{b}\right\rangle$ is the second fundamental form of $\partial \Omega$.

We first recall the following formula for Killing connections (see for example [1]):

$$
\int_{\Omega}\left(|\widehat{\nabla} \psi|^{2}+\frac{1}{4}\left(R+6 \kappa^{2}\right)|\psi|^{2}-|\widehat{D} \psi|^{2}\right)=\int_{\partial \Omega}\left\langle\psi,\left(\widehat{\nabla}_{e_{3}}+c\left(e_{3}\right) \cdot \widehat{D}\right) \psi\right\rangle .
$$

Here $\widehat{D}$ is the Killing Dirac operator, $\widehat{D} \psi=c\left(e_{i}\right) \cdot \widehat{\nabla}_{e_{i}} \psi$. The right hand side becomes

$$
\int_{\partial \Omega}\left\langle\psi, c\left(e_{3}\right) \cdot c\left(e_{a}\right) \cdot \widehat{\nabla}_{e_{a}} \psi\right\rangle
$$

We calculate using (3.1)

$$
\begin{aligned}
& c\left(e_{3}\right) \cdot c\left(e_{a}\right) \cdot \widehat{\nabla}_{e_{a}} \psi \\
& \quad=c\left(e_{3}\right) \cdot c\left(e_{a}\right) \cdot \nabla_{e_{a}}^{\partial \Omega} \psi+\frac{1}{2} h_{a b} c\left(e_{a}\right) \cdot c\left(e_{b}\right) \cdot \psi-\sqrt{-1} \kappa c\left(e_{3}\right) \cdot \psi .
\end{aligned}
$$

We recall that the Clifford multiplication on $\partial \Omega$ satisfies $c_{\partial \Omega}\left(e_{a}\right)=c\left(e_{a}\right)$. $c\left(e_{3}\right)$, and thus $c\left(e_{3}\right) \cdot c\left(e_{a}\right) \cdot \nabla_{e_{a}}^{\partial \Omega}=-D^{\partial \Omega}$, the Dirac operator on $\partial \Omega$. Also, using the property of the Clifford multiplication, it is not hard to see $h_{a b}$ $c\left(e_{a}\right) \cdot c\left(e_{b}\right)=-H$.

Proposition 3.1. Let $\left(\Omega, g_{i j}\right)$ be a compact three-manifold with boundary $\partial \Omega$, then for any spinor $\psi$ we have

$$
\begin{aligned}
& \int_{\Omega}\left(|\widehat{\nabla} \psi|^{2}+\frac{1}{4}\left(R+6 \kappa^{2}\right)|\psi|^{2}-|\widehat{D} \psi|^{2}\right) \\
& \quad=\int_{\partial \Omega}\left\langle\psi,-D^{\partial \Omega} \psi-\frac{1}{2} H \psi-\sqrt{-1} \kappa c\left(e_{3}\right) \cdot \psi\right\rangle,
\end{aligned}
$$

where $e_{3}$ is the outward normal of $\partial \Omega$ and $H=\left\langle\nabla_{e_{a}} e_{3}, e_{a}\right\rangle$ is the mean curvature.

In our situation, there are two metrics on $M=\mathbb{H}_{-\kappa^{2}}^{3} \backslash \Omega_{0}$. One is the hyperbolic metric $g^{\prime}=d r^{2}+g_{a b}(r)$, where $g_{a b}(r)$ is the induced metric on $\Sigma_{r}$ and $e^{-2 \kappa r} g_{a b}(r)$ is uniformly equivalent to the standard metric on $S^{2}$. The other metric is $g^{\prime \prime}=u^{2} d r^{2}+g_{a b}(r)$. 
$g^{\prime}$ and $g^{\prime \prime}$ induce the same metrics on $\Sigma_{r}$ while the unit normal vectors are different. They are denoted by $e_{3}^{\prime \prime}=\frac{1}{u} \frac{\partial}{\partial r}$ and $e_{3}^{\prime}=\frac{\partial}{\partial r}$ for $g^{\prime \prime}$ and $g^{\prime}$, respectively.

As in [1], we define the gauge transformation

$$
A:\left(T M, g^{\prime}\right) \longrightarrow\left(T M, g^{\prime \prime}\right)
$$

by $A\left(\frac{\partial}{\partial r}\right)=\frac{1}{u} \frac{\partial}{\partial r}$ and $A(X)=X$ for all $X \in T \Sigma_{r}$, or $A=\frac{1}{u} d u \otimes \frac{\partial}{\partial u}+e^{a} \otimes$ $e_{a} . A$ satisfies the relation

$$
g^{\prime \prime}(A(X), A(Y))=g^{\prime}(X, Y)
$$

As was remarked in [1], $A$ also defines a fiberwise isometry of the associated Riemannian spinor bundles $S\left(M, g^{\prime}\right)$ and $S\left(M, g^{\prime \prime}\right)$ and satisfies

$$
A\left(c^{\prime}(V) \cdot \psi\right)=c^{\prime \prime}(A(V)) \cdot A(\psi)
$$

where $c^{\prime}$ and $c^{\prime \prime}$ are the Clifford multiplication associated with $g^{\prime}$ and $g^{\prime \prime}$.

Denote the Riemannian connections of $g^{\prime}$ and $g^{\prime \prime}$ by $\nabla^{\prime}$ and $\nabla^{\prime \prime}$, and define a new connection $\bar{\nabla}$ by

$$
\bar{\nabla} \psi=A \nabla^{\prime}\left(A^{-1} \psi\right)
$$

$\nabla^{\prime \prime}$ and $\bar{\nabla}$ are both metric connections for $g^{\prime \prime}$, but $\bar{\nabla}$ has non-zero torsion. $\nabla^{\prime}, \nabla^{\prime \prime}$ and $\bar{\nabla}$ extend to spin connections on the corresponding spinor bundles.

Definition 3.2. $\phi_{0}^{\prime}$ is said to be a Killing spinor with respect to $\nabla^{\prime}$ if

$$
\nabla_{V}^{\prime} \phi_{0}^{\prime}=-\frac{\sqrt{-1}}{2} \kappa c^{\prime}(V) \cdot \phi_{0}^{\prime} .
$$

Proposition 3.3. Let $\left(M, g^{\prime \prime}\right)$ be as in Theorem 2.1 and $A$ be the gauge transformation defined in (3.3). Let $\phi_{0}^{\prime}$ be a Killing spinor with respect to $\nabla^{\prime}$ on $\mathbb{H}_{-\kappa^{2}}^{3}$ and $\phi_{0}=A \phi_{0}^{\prime}$. Then

$$
-D^{\Sigma_{r}} \phi_{0}=\frac{1}{2} H_{0} \phi_{0}+\sqrt{-1} \kappa c^{\prime \prime}\left(e_{3}^{\prime \prime}\right) \cdot \phi_{0}
$$


Proof. First of all, we have $\bar{\nabla}_{e_{a}} \phi_{0}=A\left(\nabla_{e_{a}}^{\prime} A^{-1} \phi_{0}\right)=A\left(\nabla_{e_{a}}^{\prime} \phi_{0}^{\prime}\right)$. Thus,

$$
\bar{\nabla}_{e_{a}} \phi_{0}=-\frac{\sqrt{-1}}{2} \kappa c^{\prime \prime}\left(e_{a}\right) \cdot \phi_{0}
$$

By definition,

$$
D^{\Sigma_{r}} \phi_{0}=-c^{\prime \prime}\left(e_{3}^{\prime \prime}\right) \cdot c^{\prime \prime}\left(e_{a}\right) \cdot \nabla_{e_{a}}^{\Sigma_{r}} \phi_{0}
$$

Now, we relate $\nabla_{e_{a}}^{\Sigma_{r}} \phi_{0}$ and $\bar{\nabla}_{e_{a}} \phi_{0}$. From the definition of the spin connection, we have,

$$
\begin{aligned}
\bar{\nabla}_{e_{a}} \phi_{0}= & \frac{1}{2} \sum_{b<c}\left\langle\bar{\nabla}_{e_{a}} e_{b}, e_{c}\right\rangle c^{\prime \prime}\left(e_{b}\right) \cdot c^{\prime \prime}\left(e_{c}\right) \cdot \phi_{0} \\
& +\frac{1}{2} \sum_{b=1}^{2}\left\langle\bar{\nabla}_{e_{a}} e_{b}, e_{3}^{\prime \prime}\right\rangle c^{\prime \prime}\left(e_{b}\right) \cdot c^{\prime \prime}\left(e_{3}^{\prime \prime}\right) \cdot \phi_{0} .
\end{aligned}
$$

Recall that the relation between $\bar{\nabla}$ and $\nabla^{\prime}$ is

$$
\bar{\nabla} \psi=A \nabla^{\prime}\left(A^{-1} \psi\right)
$$

Also $A\left(e_{3}^{\prime}\right)=e_{3}^{\prime \prime}$, and $A(X)=X$ for all $X \in T \Sigma_{r}$.

We calculate the terms in (3.7) and get $\bar{\nabla}_{e_{a}} e_{b}=A\left(\nabla_{e_{a}}^{\prime} e_{b}\right)$ and $\left\langle\bar{\nabla}_{e_{a}} e_{b}, e_{c}\right\rangle=\left\langle\nabla_{e_{a}}^{\prime} e_{b}, e_{c}\right\rangle . \quad$ Now $\left\langle\bar{\nabla}_{e_{a}} e_{b}, e_{3}^{\prime \prime}\right\rangle=\left\langle A\left(\nabla_{e_{a}}^{\prime} e_{b}\right), e_{3}^{\prime \prime}\right\rangle=\left\langle A\left(\nabla_{e_{a}}^{\prime} e_{b}\right)\right.$, $\left.A\left(e_{3}^{\prime}\right)\right\rangle=\left\langle\nabla_{e_{a}}^{\prime} e_{b}, e_{3}^{\prime}\right\rangle=-h_{a b}^{0}$. Thus

$$
\bar{\nabla}_{e_{a}} \phi_{0}=\nabla_{e_{a}}^{\Sigma_{r}} \phi_{0}-\frac{1}{2} h_{a b}^{0} c^{\prime \prime}\left(e_{b}\right) \cdot c^{\prime \prime}\left(e_{3}\right) \cdot \phi_{0} .
$$

Plug (3.8) in (3.6) and multiply by $c^{\prime \prime}\left(e_{3}^{\prime \prime}\right)$ to derive

$$
D^{\Sigma_{r}} \phi_{0}=-c^{\prime \prime}\left(e_{3}^{\prime \prime}\right) \cdot c^{\prime \prime}\left(e_{a}\right) \cdot\left[\bar{\nabla}_{e_{a}} \phi_{0}+\frac{1}{2} h_{a b}^{0} c^{\prime \prime}\left(e_{b}\right) \cdot c^{\prime \prime}\left(e_{3}^{\prime \prime}\right) \cdot \phi_{0}\right] .
$$

Plug (3.5) into (3.9), and we obtain the equality.

Definition 3.4. For any spinor field $\psi$ on $\left(M, g^{\prime \prime}\right)$, we define the mass expression to be

$$
m_{r}(\psi):=\int_{\Sigma_{r}}\left\langle\psi,-D^{\Sigma_{r}} \psi\right\rangle-\frac{1}{2} \int_{\Sigma_{r}} \mathcal{H}|\psi|_{g^{\prime \prime}}^{2}-\int_{\Sigma_{r}}\left\langle\psi, \sqrt{-1} \kappa c^{\prime \prime}\left(e_{3}^{\prime \prime}\right) \psi\right\rangle .
$$

By Proposition 3.3, we obtain 
Corollary 3.5. Let $\phi_{0}^{\prime}$ be a Killing spinor with respect to $\nabla^{\prime}$ on $\mathbb{H}_{-\kappa^{2}}^{3}$ and $\phi_{0}=A \phi_{0}^{\prime}$, then the mass expression for $\phi_{0}$ is

$$
m_{r}\left(\phi_{0}\right)=\frac{1}{2} \int_{\Sigma_{r}}\left(H_{0}-\mathcal{H}\right)\left|\phi_{0}\right|_{g^{\prime \prime}}^{2}
$$

Of course $\left|\phi_{0}\right|_{g^{\prime \prime}}^{2}=\left|A \phi_{0}^{\prime}\right|_{g^{\prime \prime}}^{2}=\left|\phi_{0}^{\prime}\right|_{g^{\prime}}^{2}$

\section{Killing spinors on hyperbolic spaces}

We first recall the model for the hyperbolic space $\mathbb{H}_{-\kappa^{2}}^{3}$ of sectional curvature $-\kappa^{2}$. Let $\mathbb{R}^{3,1}$ be the Minkowski space with the space-time coordinates

$$
\mathbf{X}=\left(x_{1}, x_{2}, x_{3}, t\right)
$$

and the Lorentz metric $d x_{1}^{3}+d x_{2}^{3}+d x_{3}^{2}-d t^{2} . \mathbb{H}_{-\kappa^{2}}^{3}$ can be identified with the space-like hypersurface

$$
\left\{\left(x_{1}, x_{2}, x_{3}, t\right) \in \mathbb{R}^{3,1} \mid x_{1}^{2}+x_{2}^{2}+x_{3}^{2}-t^{2}=-\frac{1}{\kappa^{2}}, t>0\right\} .
$$

The following parametrization using the polar coordinates $\left(r^{\prime}, \theta, \psi\right)$ on $\mathbb{R}^{3}$ is particularly useful:

$$
\begin{aligned}
\left(x_{1}, x_{2}, x_{3}, t\right)= & \frac{1}{\kappa}\left(\sinh \kappa r^{\prime} \cos \theta, \sinh \kappa r^{\prime}\right. \\
& \left.\sin \theta \cos \psi, \sinh \kappa r^{\prime} \sin \theta \sin \psi, \cosh \kappa r^{\prime}\right) .
\end{aligned}
$$

This is indeed the geodesic coordinates given by the exponential map, where $r^{\prime}$ is the geodesic distance. The induced metric in this coordinate system is then

$$
g^{\prime}=d r^{\prime 2}+\frac{\left(\sinh \kappa r^{\prime}\right)^{2}}{\kappa^{2}}\left(d \theta^{2}+\sin ^{2} \theta d \psi^{2}\right)
$$

where $d \theta^{2}+\sin ^{2} \theta d \psi^{2}$ is the standard metric on $S^{2}$ in spherical coordinates.

The future-directed unit time-like normal of $\mathbb{H}_{-\kappa^{2}}^{3}$ is then $e_{0}=\kappa \mathbf{X}$. The second fundamental form is $p=\kappa g^{\prime}$. By picking a trivialization, the space of spinor fields on $\mathbb{H}_{-\kappa^{2}}^{3}$ can be identified with the space of smooth functions 
valued in $\mathbb{C}^{2}$. A Killing spinor $\phi^{\prime}$ on $\mathbb{H}_{-\kappa^{2}}^{3}$ satisfies the equation

$$
\nabla_{V}^{\prime} \phi^{\prime}+\frac{\sqrt{-1}}{2} \kappa c^{\prime}(V) \cdot \phi^{\prime}=0 \text { for any tangent vector } V
$$

where $\nabla^{\prime}$ is the spin connection. The Killing spinors on hyperbolic spaces were studied by Baum [3]. In the $\left(r^{\prime}, \theta, \psi\right)$ coordinate system, they can be found by a calculation similar to the one in [26](the case $\kappa=1$ ). They are of the form:

$$
\phi_{0, a}^{\prime}=\left[\begin{array}{cc}
\exp \left(\frac{\kappa r^{\prime}}{2}+i \frac{\psi}{2}\right) \cos \frac{\theta}{2} & \exp \left(\frac{\kappa r^{\prime}}{2}-i \frac{\psi}{2}\right) \sin \frac{\theta}{2} \\
-\exp \left(-\frac{\kappa r^{\prime}}{2}+i \frac{\psi}{2}\right) \sin \frac{\theta}{2} & \exp \left(-\frac{\kappa r^{\prime}}{2}-i \frac{\psi}{2}\right) \cos \frac{\theta}{2}
\end{array}\right]\left[\begin{array}{l}
a_{1} \\
a_{2}
\end{array}\right]
$$

where $a=\left[\begin{array}{l}a_{1} \\ a_{2}\end{array}\right] \in \mathbb{C}^{2}$ is a constant spinor in this trivialization.

We calculate the square norm of $\phi_{0, a}^{\prime}$.

$$
\begin{aligned}
\left|\phi_{0, a}^{\prime}\right|^{2}= & \left(\left|a_{1}\right|^{2}+\left|a_{2}\right|^{2}\right) \cosh \kappa r^{\prime}+\left(\left|a_{1}\right|^{2}-\left|a_{2}\right|^{2}\right) \sinh \kappa r^{\prime} \cos \theta \\
& +\left(a_{1} \bar{a}_{2}+\bar{a}_{1} a_{2}\right) \sinh \kappa r^{\prime} \sin \theta \cos \psi \\
& +\sqrt{-1}\left(a_{1} \bar{a}_{2}-\bar{a}_{1} a_{2}\right) \sinh \kappa r^{\prime} \sin \psi \sin \theta
\end{aligned}
$$

This can be written in terms of $\mathbf{X}$ as

$$
\left|\phi_{0, a}^{\prime}\right|^{2}=-\kappa \mathbf{X} \cdot \zeta(a)
$$

where $\cdot$ is the Lorentz inner product in $\mathbb{R}^{3,1}$ and $\zeta(a) \in \mathbb{R}^{3,1}$ is (4.5)

$$
\left(-\left(\left|a_{1}\right|^{2}-\left|a_{2}\right|^{2}\right),-\left(a_{1} \bar{a}_{2}+\bar{a}_{1} a_{2}\right),-\sqrt{-1}\left(a_{1} \bar{a}_{2}-\bar{a}_{1} a_{2}\right),\left|a_{1}\right|^{2}+\left|a_{2}\right|^{2}\right) .
$$

Denote $\frac{\partial}{\partial x_{1}}=E_{1}, \frac{\partial}{\partial x_{2}}=E_{2}, \frac{\partial}{\partial x_{3}}=E_{3}$ and $\frac{\partial}{\partial t}=E_{0}$, and pick the following trivialization of the Clifford matrices for this orthonormal 
basis of $\mathbb{R}^{3,1}$ :

$$
\begin{aligned}
& c\left(E_{1}\right)=\left[\begin{array}{cc}
\sqrt{-1} & 0 \\
0 & -\sqrt{-1}
\end{array}\right], \\
& c\left(E_{2}\right)=\left[\begin{array}{cc}
0 & \sqrt{-1} \\
\sqrt{-1} & 0
\end{array}\right], \\
& c\left(E_{3}\right)=\left[\begin{array}{cc}
0 & 1 \\
-1 & 0
\end{array}\right] \\
& \text { and } c\left(E_{0}\right)=\left[\begin{array}{cc}
\sqrt{-1} & 0 \\
0 & \sqrt{-1}
\end{array}\right] .
\end{aligned}
$$

$\zeta$ can be expressed as

$$
\begin{aligned}
\zeta(a)= & \sqrt{-1}\left(\left\langle c\left(E_{1}\right) a, a\right\rangle E_{1}+\left\langle c\left(E_{2}\right) a, a\right\rangle E_{2}\right. \\
& \left.+\left\langle c\left(E_{3}\right) a, a\right\rangle E_{3}-\left\langle c\left(E_{0}\right) a, a\right\rangle E_{0}\right)
\end{aligned}
$$

where $\langle\cdot, \cdot\rangle$ is the Hermitian product on $\mathbb{C}^{2}$.

From this expression, it is clear that $\zeta(a)$ is independent of the choice of the orthonormal frames in the Minkowski space. It can be checked directly that $\zeta$ maps $\mathbb{C}^{2}$ onto the future directed light cone $C_{0}=\left\{x_{1}^{2}+x_{2}^{2}+x_{3}^{2}-\right.$ $\left.t^{2}=0, t>0\right\}$. In fact, the restriction of $\zeta$ to $S^{3} \subset \mathbb{C}^{2}$ gives the Hopf map onto $S^{2}=C_{0} \cap\{t=1\}$.

To summarize,

Proposition 4.1. For any $a=\left[\begin{array}{l}a_{1} \\ a_{2}\end{array}\right] \in \mathbb{C}^{2}$, the square norm of the Killing spinor $\phi_{0, a}^{\prime}(4.3)$ on $\mathbb{H}_{-\kappa^{2}}^{3}$ is given by

$$
\left|\phi_{0, a}^{\prime}\right|^{2}=-\kappa \mathbf{X} \cdot \zeta(a)
$$

where $\mathbf{X}=\left(x_{1}, x_{2}, x_{3}, t\right)$ is the Minkowski position vector and $\zeta$ is defined in (4.5).

In terms of the Clifford multiplication.

$$
\left|\phi_{0, a}^{\prime}\right|^{2}=-\sqrt{-1} \kappa\langle c(\mathbf{X}) a, a\rangle
$$

Denote the Hessian of $\left|\phi_{0, a}^{\prime}\right|^{2}$ on $\mathbb{H}_{-\kappa^{2}}^{3}$ by $\nabla \nabla^{-\kappa^{2}}\left|\phi_{0, a}^{\prime}\right|^{2}$. Since $\mathbf{X} \cdot \zeta(a)$ is a linear function on $\mathbb{R}^{3,1}$ and the second fundamental form of $\mathbb{H}_{-\kappa^{2}}^{3}$ is given 
by $\kappa g^{\prime}$; it is not hard to see that

$$
\nabla \nabla^{-\kappa^{2}}\left|\phi_{0, a}^{\prime}\right|^{2}=\kappa^{2}\langle\cdot, \cdot\rangle\left|\phi_{0, a}^{\prime}\right|^{2} .
$$

We calculate

$$
\Delta_{r}\left|\phi_{0, a}^{\prime}\right|^{2}=\sum_{i=1}^{2} \nabla \nabla^{-\kappa^{2}}\left|\phi_{0, a}^{\prime}\right|^{2}\left(e_{i}, e_{i}\right)-\left\langle\nabla\left|\phi_{0, a}^{\prime}\right|^{2}, \vec{H}_{0}\right\rangle,
$$

where $\vec{H}_{0}$ is the mean curvature vector of $\Sigma_{r}$.

As $\vec{H}_{0}=H_{0} \frac{\partial}{\partial r}$, we have

$$
H_{0} \frac{\partial\left|\phi_{0, a}^{\prime}\right|^{2}}{\partial r}=-\Delta_{r}\left|\phi_{0, a}^{\prime}\right|^{2}+2 \kappa^{2}\left|\phi_{0, a}^{\prime}\right|^{2} .
$$

This equation will be used to define the vector-valued function $\mathbf{W}^{0}$ in the statements of Theorem 1.3 and 1.4.

We shall express the limit of the mass expression for $\phi_{0, a}=A \phi_{0, a}^{\prime}$ :

$$
\lim _{r \rightarrow \infty} \int_{\Sigma_{r}}\left(H_{0}-\mathcal{H}\right)\left|\phi_{0, a}\right|^{2}=-\kappa \lim _{r \rightarrow \infty} \int_{\Sigma_{r}}\left(H_{0}-\mathcal{H}\right) \mathbf{X} \cdot \zeta(a)
$$

by the Gauss map of $\Sigma_{0}$.

Given a surface $F_{0}: \Sigma \rightarrow \mathbb{H}_{-\kappa^{2}}^{3}$, we consider the associated map

$$
\gamma_{0}: \Sigma \rightarrow C_{0}
$$

into the light cone by

$$
\gamma_{0}=\kappa \mathbf{X}\left(F_{0}\right)+\mathbf{N}
$$

where $\mathbf{N}$ is the normal to $\Sigma_{0}$ in $\mathbb{H}_{-\kappa^{2}}^{3}$.

It is not hard to check the image of $\gamma_{0}$ is in the light cone, and in fact the projection of $\gamma_{0}$ gives the hyperbolic Gauss map [6].

Proposition 4.2. Let $M$ be given as in the assumption of Theorem 2.1. For an asymptotically Killing spinor $\phi_{0, a}=A \phi_{0, a}^{\prime}$ on $\left(M, g^{\prime \prime}\right)$, we have

$$
\lim _{r \rightarrow \infty} \int_{\Sigma_{r}}\left(H_{0}-\mathcal{H}\right)\left|\phi_{0, a}\right|^{2}=-2 \kappa \int_{\Sigma_{\infty}} v_{\infty} \gamma_{0}(x) \cdot \zeta(a),
$$

where $\gamma_{0}$ is defined by (4.8) and $v_{\infty}$ is defined in Theorem 2.1. $\Sigma_{\infty}$ is 
$\Sigma$ equipped with the metric $g_{a b}(\infty)=\lim _{r \rightarrow \infty} e^{-2 \kappa r} g_{a b}(r)$ or the pull-back metric by $\gamma_{0}$.

Proof. By formula (2.9), we have

$$
\gamma_{0}(p)=\lim _{r \rightarrow \infty} e^{-\kappa r} \kappa \mathbf{X}(F(p, r)) .
$$

On the other hand, by Proposition 4.1, we have

$$
-\gamma_{0} \cdot \zeta(a)=\lim _{r \rightarrow \infty} e^{-\kappa r}\left|\phi_{0, a}^{\prime}\right|^{2} .
$$

The limiting metric $g_{a b}(\infty)$ is well defined by (2.5). By Proposition 4.1, we have

$$
\int_{\Sigma_{r}}\left(H_{0}-\mathcal{H}\right)\left|\phi_{0, a}\right|^{2}=-\int_{\Sigma_{r}} H_{0}\left(1-u^{-1}\right) \kappa \mathbf{X} \cdot \zeta(a) .
$$

The integrand can be regrouped as

$$
H_{0}\left(1-u^{-1}\right) \kappa \mathbf{X} \cdot \zeta(a)=H_{0}\left[e^{3 \kappa r}\left(1-u^{-1}\right)\right]\left[e^{-\kappa r} \kappa \mathbf{X} \cdot \zeta(a)\right] e^{-2 \kappa r} .
$$

The proposition now follows from (2.8), (2.16), that $\lim _{r \rightarrow \infty} H_{0}=2 \kappa$, and that the volume form of $g_{a b}(r)$ grows like $e^{2 \kappa r}$.

\section{The monotonicity formula}

In this section, we will define the function $\mathbf{W}^{0}$ found in the statements of Theorem 1.3 and 1.4. Recall we have an isometric embedding $F_{0}$ of $\Sigma$ into $\mathbb{H}_{-\kappa^{2}}^{3}$, and $\Sigma_{0}=F_{0}(\Sigma)$ has Gaussian curvature $>-\kappa^{2}$. This determines a foliation and the associated geometric quantities $g_{a b}(r), H_{0}, R^{r}$ and $\Delta_{r}$ on the leaves $\Sigma_{r}$ (see Section 2). We consider them as one-parameter families on the fixed space $\Sigma$ by the natural parametrization. The function $u$ is obtained by solving the initial value problem (2.14). $F_{0}$ also determines the map $\gamma_{0}: \Sigma \rightarrow C_{0}$ into the light cone. For any constant spinor $a \in \mathbb{C}^{2}$, $-\gamma_{0} \cdot \zeta(a)$ is a positive function defined on $\Sigma$ that satisfies (4.10).

$W$ is defined to be the solution of the following PDE:

$$
\begin{cases}\frac{H_{0}}{u} \frac{\partial W}{\partial r} & =-\Delta_{r} W+2 \kappa^{2} W \\ \lim _{r \rightarrow \infty} e^{-\kappa r} W(p, r) & =-\gamma_{0}(p) \cdot \zeta(a) .\end{cases}
$$

The equation is a backward parabolic equation. It is nevertheless solvable because the value of $W$ is prescribed at infinity. Of course the equation 
is motivated by (4.7), and $W$ plays the role of the squared norm of the Killing spinors in this case.

Lemma 5.1. Equation (5.1) has a unique positive solution $W$ on $\left(M, g^{\prime \prime}\right)$.

Proof. By Proposition 4.1 and (4.9), we can pretend $W(p, \infty)=\lim _{r \rightarrow \infty}$ $\left|\phi_{0, a}^{\prime}\right|^{2}$. To be precise, we set $\tilde{W}=e^{-\kappa r} W$. Then $\tilde{W}$ satisfies

$$
\frac{\partial}{\partial r} \tilde{W}=-\frac{u}{H_{0}} \Delta_{r} \tilde{W}+\kappa^{2}\left(\frac{2 u}{H_{0}}-\frac{1}{\kappa}\right) \tilde{W} .
$$

Recall that $\tilde{\Delta}_{r}=e^{2 \kappa r} \Delta_{r}$ is the Laplace operator of the rescaled metric $\tilde{g}_{a b}$ which is bounded, and thus

$$
2 e^{2 \kappa r} \frac{\partial}{\partial r} \tilde{W}=-\frac{2 u}{H_{0}} \tilde{\Delta}_{r} \tilde{W}+2 \kappa^{2} e^{2 \kappa r}\left(\frac{2 u}{H_{0}}-\frac{1}{\kappa}\right) \tilde{W} .
$$

By (2.13) and (2.8), we have $\left|\frac{2 u}{H_{0}}-\frac{1}{\kappa}\right|<C e^{-3 \kappa r}$. We reparametrize this equation by taking $\tau=\frac{1}{4 \kappa} e^{-2 \kappa r}$. Then $2 e^{2 \kappa r} \frac{\partial}{\partial r}=-\frac{\partial}{\partial \tau}$; and the equation becomes

$$
\begin{cases}\frac{\partial}{\partial \tau} \tilde{W} & =\frac{2 u}{H_{0}} \tilde{\Delta}_{r} \tilde{W}-2 \kappa^{2} e^{2 \kappa r}\left(\frac{2 u}{H_{0}}-\frac{1}{\kappa}\right) \tilde{W} \\ \tilde{W}(\cdot, \tau=0) & =-\gamma_{0} \cdot \zeta(a) .\end{cases}
$$

This is now a forward linear parabolic equation for $\tau=0(r=\infty)$ to $\tau=\frac{1}{4 \kappa}(r=0)$. Since $\tilde{W}>0$ at $\tau=0$, it remains positive by the maximum principle. Thus, $W$ is positive as well.

Now we prove a monotonicity formula that generalizes the one in [18]:

Proposition 5.2. Let $M$ be given as in the assumption of Theorem 2.1. For any $W$ satisfying (5.1), the quantity

$$
m_{W}(r)=\int_{\Sigma_{r}}\left(H_{0}-\mathcal{H}\right) W
$$

is monotone decreasing in $r$, and

$$
\lim _{r \rightarrow \infty} \int_{\Sigma_{r}}\left(H_{0}-\mathcal{H}\right) W=\lim _{r \rightarrow \infty} \int_{\Sigma_{r}}\left(H_{0}-\mathcal{H}\right)\left|\phi_{0, a}\right|^{2} .
$$


Proof. Recall that $\mathcal{H}=\frac{H_{0}}{u}$. We compute

$$
\begin{aligned}
\frac{d}{d r} m_{W}= & \int_{\Sigma_{r}} \frac{\partial H_{0}}{\partial r}\left(1-u^{-1}\right) W+H_{0} u^{-2} \frac{\partial u}{\partial r} \mu \\
& +H_{0}\left(1-u^{-1}\right) \frac{d W}{d r}+\left(H_{0}\right)^{2}\left(1-u^{-1}\right) W
\end{aligned}
$$

Plugging in Equation (2.14) and integrating by parts, we obtain

$$
\begin{aligned}
& \int_{S_{r}}\left(\frac{\partial H_{0}}{\partial r}+H_{0}^{2}\right)\left(1-u^{-1}\right) W+\frac{1}{2}\left(u^{-1}-u\right)\left(R^{r}+6 \kappa^{2}\right) W \\
& +H_{0}\left(1-u^{-1}\right) \frac{d W}{d r}+\int(u-1) \Delta_{r} W
\end{aligned}
$$

The Gauss formula says

$$
R^{r}=-2 \kappa^{2}+\left|H_{0}\right|^{2}-|A|^{2}
$$

Combine this with Equation (2.4) to obtain

$$
\frac{\partial H_{0}}{\partial r}+H_{0}^{2}=R^{r}+4 \kappa^{2}
$$

We check the following identity holds:

$$
\begin{gathered}
\left(R^{r}+4 \kappa^{2}\right)\left(1-u^{-1}\right)+\frac{1}{2}\left(u^{-1}-u\right)\left(R^{r}+6 \kappa^{2}\right) \\
=-\frac{1}{2} u^{-1}(u-1)^{2}\left(R^{r}+2 \kappa^{2}\right)-2 \kappa^{2}(u-1)
\end{gathered}
$$

and thus

$$
\begin{aligned}
\frac{d m}{d r}= & -\frac{1}{2} \int u^{-1}(u-1)^{2}\left(R^{r}+2 \kappa^{2}\right) W \\
& +\int(u-1)\left(\frac{H_{0}}{u} \frac{d W}{d r}+\Delta_{r} W-2 \kappa^{2} W\right)
\end{aligned}
$$

The second term on the right hand side vanishes by (5.1). Our assumption implies $R^{r}>-2 \kappa^{2}$, and thus $\frac{d m}{d r} \leq 0$. 
By the prescribed value of (5.1) at $\infty$,

$$
\begin{aligned}
\lim _{r \rightarrow \infty} \int_{\Sigma_{r}}\left(H_{0}-\mathcal{H}\right) W & =\lim _{r \rightarrow \infty} \int_{\Sigma_{r}} H_{0}\left(1-u^{-1}\right) e^{3 \kappa r}\left(e^{-\kappa r} W\right) e^{2 \kappa r} \\
& =-\int_{\Sigma_{\infty}} 2 \kappa v_{\infty} \gamma_{0} \cdot \zeta(a)
\end{aligned}
$$

By Proposition 4.2, this equals

$$
\frac{1}{\kappa} \lim _{r \rightarrow \infty} \int_{\Sigma_{r}}\left(H_{0}-\mathcal{H}\right)\left|\phi_{0, a}\right|^{2}
$$

Since the Equation (5.1) is linear, we may as well consider a four-vector valued function $\mathbf{W}: \Sigma \times[0, \infty) \rightarrow \mathbb{R}^{3,1}$ that satisfies

$$
\begin{cases}\frac{H_{0}}{u} \frac{d \mathbf{W}}{d r} & =-\Delta_{r} \mathbf{W}+2 \kappa^{2} \mathbf{W} \\ \lim _{r \rightarrow \infty} e^{-\kappa r} \mathbf{W}(p, r) & =-\gamma_{0}(p)\end{cases}
$$

Set $W=\mathbf{W} \cdot \zeta(a)$. We obtain the following.

Proposition 5.3. Let $M$ be given as in the assumption of Theorem 2.1. If $\mathbf{W}$ satisfies (5.3), the quantity

$$
\int_{\Sigma_{r}}\left(H_{0}-\mathcal{H}\right) \mathbf{W} \cdot \zeta(a)
$$

is monotone decreasing in $r$ for any $\zeta(a)$, and

$$
\lim _{r \rightarrow \infty} \int_{\Sigma_{r}}\left(H_{0}-\mathcal{H}\right) \mathbf{W} \cdot \zeta(a)=\lim _{r \rightarrow \infty} \int_{\Sigma_{r}}\left(H_{0}-\mathcal{H}\right)\left|\phi_{0, a}\right|^{2} .
$$

We notice that $\gamma_{0}=\lim _{r \rightarrow \infty} e^{-\kappa r} \kappa \mathbf{X}$ is future-directed time-like and $-\gamma_{0} \cdot \zeta(a) \geq 0$ for any $\zeta(a)$. By the maximum principle and the following characterization of future-directed time-like vectors, $\mathbf{W}(r)$ remains a past-directed time-like vector.

Lemma 5.4. A four-vector $v=\left(a_{1}, a_{2}, a_{3}, b\right)$ is future-directed time-like (non-space-like) if and only if $v \cdot \zeta<0(\leq 0)$ for all $\zeta=\left(y_{1}, y_{2}, y_{3}, 1\right)$ with $y_{1}^{2}+y_{2}^{2}+y_{3}^{2}=1$. 


\section{Positivity of the mass expression}

Given a convex isometric embedding $F_{0}: \Sigma \rightarrow \mathbb{H}_{-\kappa^{2}}^{3}$ and a function $\mathcal{H}(0)$ defined on $\Sigma$, we constructed an asymptotically hyperbolic metric $g^{\prime \prime}=$ $u^{2} d r^{2}+g_{a b}(r)$ on $M=\mathbb{H}_{-\kappa^{2}}^{3} \backslash \Omega_{0}$, where $g_{a b}(r)$ is the induced metric on the leaves $\Sigma_{r}$ and $\mathcal{H}(r)$ is the mean curvature of $\Sigma_{r}$ with respect to the outward normal in $\left(M, g^{\prime \prime}\right)$. Recall for each Killing spinor $\phi_{a, 0}^{\prime}$ on $\mathbb{H}_{-\kappa^{2}}^{3}$, we obtained an asymptotic Killing spinor $\phi_{a, 0}=A \phi_{a, 0}^{\prime}$ on $\left(M, g^{\prime \prime}\right)$. In this section, we prove the mass expression

$$
\lim _{r \rightarrow \infty} \frac{1}{2} \int_{\Sigma_{r}}\left(H_{0}-\mathcal{H}\right)\left|\phi_{a, 0}\right|^{2}
$$

in Proposition 5.2 is positive under certain assumptions on $\mathcal{H}$.

For a suitable chosen $\mathcal{H}(0)$, we prove there exists a Killing-harmonic spinor $\phi_{a}$ on $\left(M, g^{\prime \prime}\right), \widehat{D} \phi_{a}=0$, with the appropriate asymptotic behavior to assure that

$$
\lim _{r \rightarrow \infty} \int_{\Sigma_{r}}\left\langle\phi_{a},\left(\widehat{\nabla}_{\nu_{r}}+c^{\prime \prime}\left(\nu_{r}\right) \cdot \widehat{D}\right) \phi_{a}\right\rangle=\lim _{r \rightarrow \infty} \int_{\Sigma_{r}}\left\langle\phi_{a, 0},\left(\widehat{\nabla}_{\nu_{r}}+c^{\prime \prime}\left(\nu_{r}\right) \cdot \widehat{D}\right) \phi_{a, 0}\right\rangle .
$$

The left hand side can be shown to be non-negative by the SchrödingerLichnerowciz formula for harmonic-Killing spinors. Now by Corollary 3.5, the right hand side is the mass expression $\lim _{r \rightarrow \infty} \int_{\Sigma_{r}}\left(H_{0}-\mathcal{H}\right)\left|\phi_{a, 0}\right|_{g^{\prime \prime}}^{2}$

Since the metric $g^{\prime \prime}$ depends on the embedding $F_{0}$ and $\mathcal{H}(0)$, the question is now: For what kind of $\left(F_{0}(\Sigma), \mathcal{H}(0)\right)$ can we fill in $\left(M, g^{\prime \prime}\right)$ with a compact three-manifold $\Omega$ with boundary so that the resulting manifold has positive total mass.

\subsection{Riemannian version}

The following theorem is a generalization of Shi-Tam [18] which corresponds to the case when $\kappa=0$.

Theorem 6.1. For $\kappa>0$, let $\Omega$ be a compact three-manifold with smooth boundary $\partial \Omega=\Sigma$ and with scalar curvature $R \geq-6 \kappa^{2}$. Suppose $\Sigma$ has positive mean curvature $H$ with respect to the outward normal and has sectional curvature $K>-\kappa^{2}$. Let $F_{0}$ be the isometric embedding of $\Sigma$ into $\mathbb{H}_{-\kappa^{2}}^{3}$ and $\Omega_{0}$ be the region in $\mathbb{H}_{-\kappa^{2}}^{3}$ enclosed by $F_{0}(\Sigma)$. Suppose $M=\mathbb{H}_{-\kappa^{2}}^{3} \backslash \Omega_{0}$ is equipped with the metric $g^{\prime \prime}=u^{2} d r^{2}+g_{a b}(r)$ so that $u$ satisfies (2.14) with $\mathcal{H}(0)=H$. Let $\tilde{M}=M \cup_{F_{0}} \Omega$ be equipped with the metric $\tilde{g}_{i j}$ such 
that $\tilde{g}_{i j}=g_{i j}$ on $\Omega$ and $\tilde{g}_{i j}=g_{i j}^{\prime \prime}$ on $M$. Let $\widehat{\nabla}_{V}=\tilde{\nabla}_{V}+\frac{\sqrt{-1}}{2} \kappa \tilde{c}(V)$ be the Killing connection associated with $\tilde{g}_{i j}$ and $\widehat{D}=\tilde{c}\left(e_{i}\right) \cdot \widehat{\nabla}_{e_{i}}$ the Killing-Dirac operator. Then for each Killing spinor $\phi_{0, a}^{\prime}$ on $\mathbb{H}_{-\kappa^{2}}^{3}$, there exists a Killingharmonic spinor $\phi_{a}, \widehat{D} \phi_{a}=0$, on $\tilde{M}$ that is asymptotic to $\phi_{0, a}=A \phi_{0, a}^{\prime}$ in the sense of (6.1).

Proof. We remark that the resulting metric $\tilde{g}$ is Lipschitz and $R \geq-6 \kappa^{2}$ holds on $\left(\tilde{M} \backslash \partial \Omega, \tilde{g}_{i j}\right)$.

Notice that we can choose a smooth structure (coordinates) near the joint $\partial \Omega$ so the coefficients $\tilde{g}_{i j}$ are Lipschitz functions (see Liu-Yau [12] Section 4.5). In the following, we denote by $L^{2}$ and $W^{1,2}$, the space of $L^{2}$ and $W^{1,2}$ sections of the spinor bundle $S(\tilde{M}, \tilde{g})$ as the completion of $C^{\infty}$ sections of compact support with respect to the smooth structure and the corresponding norms.

For a Killing spinor $\phi_{a, 0}^{\prime}$ on $\mathbb{H}_{-\kappa^{2}}^{3}$, we obtain an asymptotic Killing spinor $\phi_{a, o}=A \phi_{a, 0}^{\prime}$ on $\left(M, g^{\prime \prime}\right)$. We can multiply $\phi_{a, 0}$ by a cut-off function $f$ such that $f \phi_{a, 0}$ is a smooth spinor defined on $\tilde{M}$. By abusing notation, we still denote this spinor on $\tilde{M}$ by $\phi_{a, 0}$.

Near infinity with respect to the connection $\bar{\nabla}=A \nabla^{\prime} A^{-1}, \phi_{a, 0}$ satisfies $\bar{\nabla}_{e_{b}} \phi_{a, 0}=-\frac{\sqrt{-1}}{2} \kappa \tilde{c}\left(e_{b}\right) \cdot \phi_{a, 0}$ by (3.5). On the other hand, $\bar{\nabla}_{\frac{\partial}{\partial r}} \phi_{a, 0}=$ $-\frac{\sqrt{-1}}{2} \frac{\kappa}{u} \tilde{c}\left(\frac{\partial}{\partial r}\right) \phi_{a, 0}$.

We compute

$$
\widehat{\nabla}_{e_{b}} \phi_{a, 0}=\tilde{\nabla}_{e_{b}} \phi_{a, 0}+\frac{\sqrt{-1}}{2} \kappa \tilde{c}\left(e_{b}\right) \cdot \phi_{a, 0}=\tilde{\nabla}_{e_{b}} \phi_{a, 0}-\bar{\nabla}_{e_{b}} \phi_{a, 0}
$$

and

$$
\widehat{\nabla}_{\frac{\partial}{\partial r}} \phi_{a, 0}=\tilde{\nabla}_{\frac{\partial}{\partial r}} \phi_{a, 0}-u \bar{\nabla}_{\frac{\partial}{\partial r}} \phi_{a, 0} .
$$

The difference of these two connections is estimated in Lemma 2.1 in [1], and

$$
\left|\widehat{\nabla} \phi_{a, 0}\right|_{\tilde{g}} \leq C\left|A^{-1}\right|_{\tilde{g}}\left|\nabla^{\prime} A\right|_{\tilde{g}}\left|\phi_{a, 0}\right|_{\tilde{g}}
$$

Since $A=\frac{1}{u} d u \otimes \frac{\partial}{\partial u}+e^{a} \otimes e_{a}$ and $u$ and its derivatives are estimated by (2.13), we have $A^{-1}$ is bounded and $\nabla^{\prime} A=O\left(e^{-3 \kappa r}\right)$. Also $\left|\phi_{a, 0}\right|^{2}=O\left(e^{\kappa r}\right)$. Thus $\left|\widehat{\nabla} \phi_{a, 0}\right| \leq C e^{-(5 / 2) \kappa r}$. Since the volume element of $\left(M, g^{\prime \prime}\right)$ is $u \sqrt{\operatorname{det} g_{a b}}$ and $\sqrt{\operatorname{det} g_{a b}}$ is of the order $e^{2 \kappa r}$ (see (2.5)), both $\widehat{D} \phi_{a, 0}$ and $\widehat{\nabla} \phi_{a, 0}$ are in $L^{2}$. 
We shall prove there exists a $\phi_{1} \in W^{1,2}$ such that

$$
\widehat{D} \phi_{1}=-\widehat{D} \phi_{a, 0}
$$

This is done by showing that the map $\widehat{D}: W^{1,2} \rightarrow L^{2}$ is surjective.

We need the following relations which are easy to derive:

$$
\begin{aligned}
|\widehat{\nabla} \psi|^{2} & =|\tilde{\nabla} \psi|^{2}+\frac{3 \kappa^{2}}{4}|\psi|^{2}+\frac{\sqrt{-1} \kappa}{2} e_{i}\left\langle\tilde{c}\left(e_{i}\right) \psi, \psi\right\rangle, \\
|\widehat{D} \psi|^{2} & =|\tilde{D} \psi|^{2}+\frac{9 \kappa^{2}}{4}|\psi|^{2}+\frac{3 \sqrt{-1} \kappa}{2} e_{i}\left\langle\tilde{c}\left(e_{i}\right) \psi, \psi\right\rangle, \\
e_{i}\left\langle\tilde{c}\left(e_{i}\right) \psi, \psi\right\rangle & =\langle\tilde{D} \psi, \psi\rangle-\langle\psi, \tilde{D} \psi\rangle .
\end{aligned}
$$

We proceed as in Lemma 4.4 and Proposition 3.3 of [1]. Define the functional

$$
l(\psi)=\int_{\tilde{M}}\left\langle\widehat{D} \psi, \widehat{D} \phi_{a, 0}\right\rangle
$$

on $W^{1,2}$.

Since $\widehat{D} \phi_{a, 0} \in L^{2}$, this functional is bounded on $W^{1,2}$. Define the sesquilinear form

$$
B(\psi, \phi)=\int_{\tilde{M}}\langle\widehat{D} \psi, \widehat{D} \phi\rangle
$$

We shall show $B$ is bounded and coercive on $W^{1,2}$. Then by Lax-Milgram, there exists a $\phi_{1} \in W^{1,2}$ such that for all $\psi \in W^{1,2}$ we have

$$
B\left(\psi, \phi_{1}\right)=\int_{\tilde{M}}\left\langle\widehat{D} \psi, \widehat{D} \phi_{1}\right\rangle=-\int_{\tilde{M}}\left\langle\widehat{D} \psi, \widehat{D} \phi_{a, 0}\right\rangle .
$$

To see that $B$ is bounded, recall on $\Omega$ we have,

$$
\begin{aligned}
& \int_{\Omega}\left(|\widehat{\nabla} \psi|^{2}+\frac{1}{4}\left(R+6 \kappa^{2}\right)|\psi|^{2}-|\widehat{D} \psi|^{2}\right) \\
& \quad=\int_{\partial \Omega}\left\langle\psi,-D^{\partial \Omega} \psi-\frac{1}{2} H \psi-\sqrt{-1} \kappa c(\nu) \cdot \psi\right\rangle,
\end{aligned}
$$

where $\nu$ is the outward normal of $\Omega$ and $-D^{\partial \Omega}=c(\nu) \cdot c\left(e_{a}\right) \nabla_{e_{a}}^{\partial \Omega} \psi$. 
Let $\tilde{M}_{r} \subset \tilde{M}$ be the region with $\partial \tilde{M}_{r}=\Sigma_{r}$. On $\tilde{M}_{r} \backslash \Omega$, where the scalar curvature $R=-6 \kappa^{2}$, we have

$$
\begin{aligned}
\int_{\tilde{M}_{r} \backslash \Omega}\left(|\widehat{\nabla} \psi|^{2}-|\widehat{D} \psi|^{2}\right)= & \int_{\partial \Omega}\left\langle\psi, D^{\partial \Omega} \psi+\frac{1}{2} H \psi+\sqrt{-1} \kappa c^{\prime \prime}(\nu) \cdot \psi\right\rangle \\
& +\int_{\Sigma_{r}}\left\langle\left(\widehat{\nabla}_{\nu_{r}}+c^{\prime \prime}\left(\nu_{r}\right) \widehat{D}\right) \psi, \psi\right\rangle
\end{aligned}
$$

where $\nu_{r}$ is the outward normal of $\Sigma_{r}$. As the mean curvatures coincide along the boundary, adding these up we obtain

$$
\int_{\tilde{M}_{r}}|\widehat{\nabla} \psi|^{2}-|\widehat{D} \psi|^{2}+\frac{1}{4} \int_{\Omega}\left(R+6 \kappa^{2}\right)|\psi|^{2}=\int_{\Sigma_{r}}\left\langle\left(\widehat{\nabla}_{\nu_{r}}+c^{\prime \prime}\left(\nu_{r}\right) \widehat{D}\right) \psi, \psi\right\rangle .
$$

By assumption $R$ is bounded. This shows $B(\psi, \psi) \leq C|\psi|_{W^{1,2}(\tilde{M})}^{2}$ for any $\psi \in C_{c}^{\infty}$. This holds for any $\psi \in W^{1,2}$, and the map $B$ is bounded.

On the other hand, since $R+6 \kappa^{2} \geq 0$ on $\Omega$,

$$
\int_{\tilde{M}_{r}}|\widehat{\nabla} \psi|^{2} \leq \int_{\tilde{M}_{r}}|\widehat{D} \psi|^{2}
$$

for any $\psi \in C_{c}^{\infty}(\tilde{M}, S)$. By $(6.2)$, this implies

$$
|\psi|_{1,2}^{2} \leq C \int_{M}|\widehat{D} \psi|^{2}
$$

for any $\psi \in W^{1,2}$.

Since $B$ is bounded and coercive on $W^{1,2}$, by Lax-Milgram, there exists a $\phi_{1} \in W^{1,2}$ such that for all $\psi \in W^{1,2}$ we have (6.3). Thus, $\phi_{a}=\phi_{1}+\phi_{a, 0}$ satisfies $\int_{\tilde{M}}\left\langle\widehat{D} \psi, \widehat{D} \phi_{a}\right\rangle=0$ for all $\psi \in W^{1,2}$.

Set $\Phi=\widehat{D} \phi_{a} . \Phi \in L^{2}$ and $\int_{\tilde{M}}\langle\widehat{D} \psi, \Phi\rangle=0$ for any $\psi \in W^{1,2}$. Integrating by parts,

$$
\int_{\tilde{M}}\langle\widehat{D} \psi, \Phi\rangle=\int_{\tilde{M}}\langle\psi,(\widehat{D}+3 \sqrt{-1} \kappa) \Phi\rangle,
$$

for any $\psi$ with compact support. This implies

$$
\widehat{D} \Phi+3 \sqrt{-1} \kappa \Phi=0
$$

weakly. Following Liu-Yau [12], we can find a coordinate system and a smooth operator $D^{\prime}$ so that $D^{\prime} \Phi=f \Phi$ and $f$ is continuous. Therefore, 
$\Phi \in W^{1, p}$ near $\partial \Omega$ for $p \geq 2$, and $\Phi \in C^{\infty}$ elsewhere. As $\widehat{D} \Phi=-3 \sqrt{-1} \kappa \Phi$, $\widehat{D} \Phi$ is in $W^{1, p}$ as well. Consider

$$
\begin{aligned}
\int_{\tilde{M}}\left\langle\widehat{D}\left(\eta^{2} \Phi\right), \widehat{D} \Phi\right\rangle & =\int_{\tilde{M}}\left\langle\eta^{2} \Phi,(\widehat{D}+3 \sqrt{-1} \kappa) \widehat{D} \Phi\right\rangle \\
& =\int_{\tilde{M}}\left\langle\eta^{2} \Phi, \widehat{D}(\widehat{D}+3 \sqrt{-1} \kappa) \Phi\right\rangle=0 .
\end{aligned}
$$

Take $\eta$ to be a cut-off function with $|\nabla \eta| \leq \frac{1}{r}$. We show that

$$
\int_{\tilde{M}_{r}}|\widehat{D} \Phi|^{2} \leq \frac{C}{r^{2}} \int_{\tilde{M}_{r}}|\Phi|^{2}
$$

Take $r \rightarrow \infty$. We obtain $\widehat{D} \Phi=0$ and together with $\widehat{D} \Phi=-3 \sqrt{-1} \kappa \Phi$, we deduce $\Phi=0$ or $\widehat{D} \phi_{a}=0$, i.e., $\phi_{a}$ is a Killing-harmonic spinor.

To prove that $\phi_{a}$ has the desired asymptotic behavior, set $\widehat{B}=\widehat{\nabla}_{\nu_{r}}+$ $\tilde{c}\left(\nu_{r}\right) \cdot \widehat{D}$. Then $\widehat{B}$ is self-adjoint on $\Sigma_{r}$. We write

$$
\begin{aligned}
& \int_{\Sigma_{r}}\left\langle\left(\widehat{\nabla}_{\nu_{r}}+c^{\prime \prime}\left(\nu_{r}\right) \widehat{D}\right) \phi_{a}, \phi_{a}\right\rangle \\
& \quad=\int_{\Sigma_{r}}\left\langle\widehat{B} \phi_{a, 0}, \phi_{a, 0}\right\rangle+\int_{\Sigma_{r}}\left\langle\widehat{B} \phi_{1}, \phi_{1}\right\rangle+\int_{\Sigma_{r}}\left\langle\widehat{B} \phi_{a, 0}, \phi_{1}\right\rangle+\int_{\Sigma_{r}}\left\langle\phi_{1}, \widehat{B} \phi_{a, 0}\right\rangle .
\end{aligned}
$$

Since $\widehat{\nabla} \phi_{a, 0} \in L^{2}, \phi_{1} \in W^{1,2}$ and $g^{\prime \prime}$ is of the form $u^{2} d r^{2}+g_{a b}(r)$ with $u \rightarrow 1$ uniformly, the last three terms all approach zero as $r \rightarrow \infty$.

\subsection{General case}

Let $\left(\Omega, g_{i j}, p_{i j}\right)$ be a compact initial data set. Suppose the boundary of $\Omega$ is a smooth surface $\Sigma$ with Gaussian curvature $K$ and mean curvature $H$ with respect to the outward normal. We assume the mean curvature vector of $\Sigma$ is space-like or $H>\left|\operatorname{tr}_{\Sigma} p\right|$.

Let $\bar{g}_{i j}=g_{i j}+f_{i} f_{j}$ be the metric on $\Omega$ from the solution of the Jang's equation with $f \equiv 1$ on $\Sigma$. For any $\kappa>0$ satisfying $K>-\kappa^{2}$, let $F$ be the isometric embedding of $\Sigma$ into $\mathbb{H}_{-\kappa^{2}}^{3} \subset \mathbb{R}^{3,1}$ and $\Omega$ be the region in $\mathbb{H}_{-\kappa^{2}}^{3}$ enclosed by $F(\Sigma)$. Suppose $M=\mathbb{H}_{-\kappa^{2}}^{3} \backslash \Omega$ is equipped with the metric $g^{\prime \prime}=$ $u^{2} d r^{2}+g_{i j}(r)$ so that $u$ satisfies $(2.14)$ with $\mathcal{H}(p)=\sqrt{H^{2}-\left(\operatorname{tr}_{\Sigma} p\right)^{2}}$. Let $\tilde{M}=M \cup_{F} \Omega$ be equipped with the metric $\tilde{g}_{i j}$ such that $\tilde{g}_{i j}=\bar{g}_{i j}$ on $\Omega$ and 
$\tilde{g}_{i j}=g_{i j}^{\prime \prime}$ on $M$. Define the Killing spin connection $\widehat{\nabla}$ by

$$
\widehat{\nabla}_{e_{i}}=\bar{\nabla}_{e_{i}}+\frac{\sqrt{-1}}{2} \kappa \bar{c}\left(e_{i}\right)
$$

on $(\Omega, \bar{g})$ and

$$
\widehat{\nabla}_{e_{i}}=\nabla_{e_{i}}^{\prime \prime}+\frac{\sqrt{-1}}{2} \kappa c^{\prime \prime}\left(e_{i}\right)
$$

on $\left(M, g^{\prime \prime}\right)$.

The associated Dirac operator is then

$$
\widehat{D}=\bar{D}-\frac{3 \sqrt{-1}}{2} \kappa
$$

on $(\Omega, \bar{g})$ and

$$
\widehat{D}=D^{\prime \prime}-\frac{3 \sqrt{-1}}{2} \kappa
$$

on $\left(M, g^{\prime \prime}\right)$.

Theorem 6.2. Under the above assumption, for each Killing spinor $\phi_{0, a}^{\prime}$ on $\mathbb{H}_{-\kappa^{2}}^{3}$, there exists a Killing-harmonic spinor $\phi_{a}, \widehat{D} \phi_{a}=0$ on $\tilde{M}$ and is asymptotic to $\phi_{0, a}=A \phi_{0, a}^{\prime}$ in the sense of (6.1).

Proof. Recall on the solution of the Jang's equation $\left(\Omega, \bar{g}_{i j}\right)$, the scalar curvature $\bar{R}$ satisfies

$$
\bar{R} \geq 2|X|^{2}-2 \operatorname{div} X
$$

On the other hand, if we denote the outward normal to $\Omega$ by $\bar{\nu}$ and the mean curvature by $\bar{H}=\left\langle\nabla_{e_{a}} \bar{\nu}, e_{a}\right\rangle$, then by Lemma 4 in [12],

$$
\bar{H}-\langle X, \bar{\nu}\rangle \geq \sqrt{H^{2}-\left(\operatorname{tr}_{\Sigma} p\right)^{2}} .
$$

We have on $\Omega$

$$
\begin{aligned}
& \int_{\Omega}|\widehat{\nabla} \psi|^{2}+\frac{1}{4} \int_{\Omega}\left(\bar{R}+6 \kappa^{2}\right)|\psi|^{2}-\int_{\Omega}|\widehat{D} \psi|^{2} \\
& =\int_{\partial \Omega}\left\langle\psi,\left(\bar{\nabla}_{\bar{\nu}}+\bar{c}(\bar{\nu}) \cdot \bar{D}\right) \psi\right\rangle+\sqrt{-1} \kappa\langle\psi, \bar{c}(\bar{\nu}) \psi\rangle .
\end{aligned}
$$

Integrating by parts, we get

$$
\frac{1}{2} \int_{\partial \Omega}\langle X, \bar{\nu}\rangle|\psi|^{2}=\frac{1}{2} \int_{\Omega} \operatorname{div} X|\psi|^{2}+\frac{1}{2} \int_{\Omega} X\left(|\psi|^{2}\right) .
$$


Formula (6.7) is equivalent to

$$
\begin{gathered}
\int_{\Omega}|\widehat{\nabla} \psi|^{2}+\frac{1}{4} \int_{\Omega}\left(\bar{R}+6 \kappa^{2}+2 \operatorname{div} X\right)|\psi|^{2}+\frac{1}{2} \int_{\Omega} X\left(|\psi|^{2}\right)-\int_{\Omega}|\widehat{D} \psi|^{2} \\
=\int_{\partial \Omega}\left\langle\psi,\left(\bar{\nabla}_{\bar{\nu}}+\bar{c}(\bar{\nu}) \cdot \bar{D}\right) \psi\right\rangle+\frac{1}{2} \int_{\partial \Omega}\langle X, \bar{\nu}\rangle|\psi|^{2}+\sqrt{-1} \kappa\langle\psi, \bar{c}(\bar{\nu}) \psi\rangle .
\end{gathered}
$$

The boundary term can be written as

$$
\int_{\partial \Omega}\left\langle\psi,-D^{\partial \Omega} \psi-\frac{1}{2} \bar{H} \psi+\frac{1}{2}\langle X, \bar{\nu}\rangle \psi-\sqrt{-1} \kappa \bar{c}(\bar{\nu}) \cdot \psi\right\rangle,
$$

where $-D^{\partial \Omega} \psi=\bar{c}(\bar{\nu}) \cdot \bar{c}\left(e_{a}\right) \cdot \nabla_{e_{a}}^{\partial \Omega} \psi$.

Let $\tilde{M}_{r} \subset \tilde{M}$ be the region with $\partial \tilde{M}_{r}=\Sigma_{r}$. On $\tilde{M}_{r} \backslash \Omega$, we have

$$
\begin{aligned}
\int_{\tilde{M}_{r} \backslash \Omega}\left(|\widehat{\nabla} \psi|^{2}-|\widehat{D} \psi|^{2}\right)= & \int_{\partial \Omega}\left\langle\psi, D^{\partial \Omega} \psi+\frac{1}{2} \mathcal{H}(0) \psi+\sqrt{-1} \kappa c^{\prime \prime}(\bar{\nu}) \cdot \psi\right\rangle \\
& +\int_{\Sigma_{r}}\left\langle\left(\widehat{\nabla}_{\nu_{r}}+c^{\prime \prime}\left(\nu_{r}\right) \cdot \widehat{D}\right) \psi, \psi\right\rangle .
\end{aligned}
$$

Adding these up, we obtain

$$
\begin{aligned}
\int_{\tilde{M}_{r}} & |\widehat{\nabla} \psi|^{2}+\frac{1}{4} \int_{\Omega}\left(\bar{R}+6 \kappa^{2}+2 \operatorname{div} X\right)|\psi|^{2}+\frac{1}{2} \int_{\Omega} X\left(|\psi|^{2}\right) \\
= & \int_{\tilde{M}_{r}}|\widehat{D} \psi|^{2}+\int_{\partial \Omega} \frac{1}{2}\left[\sqrt{H^{2}-\left(\operatorname{tr}_{\Sigma} p\right)^{2}}-(\bar{H}-\langle X, \bar{\nu}\rangle)\right]|\psi|^{2} \\
& +\int_{\Sigma_{r}}\left\langle\left(\widehat{\nabla}_{\nu_{r}}+c^{\prime \prime}\left(\nu_{r}\right) \cdot \widehat{D}\right) \psi, \psi\right\rangle .
\end{aligned}
$$

Applying this to $\psi \in C_{c}^{\infty}$, the last term vanishes. Since $\sqrt{H^{2}-\left(t r_{\Sigma} p\right)^{2}}-$ $(\bar{H}-\langle X, \nu\rangle)$ is bounded, the right hand side is bounded by $\int_{\Sigma}|\psi|^{2}$. The Sobolev trace map $W^{1,2}(\Omega) \rightarrow L^{2}(\partial \Omega)$ is bounded (see, for example, Theorem 9 of Liu-Yau [12]). Thus,

$$
\int_{\partial \Omega}|\psi|^{2} \leq C|\psi|_{W^{1,2}(\Omega)}^{2}
$$

Define $B$ as in the proof of Theorem 6.1 , we see $B$ is bounded. 
To prove $B$ is coercive on $W^{1,2}$, we assume $\psi \in C_{0}^{\infty}$ so that the boundary term on $\Sigma_{r}$ vanishes for $r$ large. By (6.5), (6.6), and (6.9),

$$
\begin{aligned}
\int_{\tilde{M}_{r}}|\widehat{D} \psi|^{2} & \geq \int_{\tilde{M}_{r}}|\widehat{\nabla} \psi|^{2}+\frac{1}{4} \int_{\Omega}\left(2|X|^{2}+6 \kappa^{2}\right)|\psi|^{2}+\frac{1}{2} \int_{\Omega} X\left(|\psi|^{2}\right) \\
& \geq \frac{1}{3} \int_{\tilde{M}_{r}}|\widehat{\nabla} \psi|^{2}+\mathfrak{R},
\end{aligned}
$$

where

$$
\mathfrak{R}=\int_{\Omega}\left[\frac{2}{3}|\widehat{\nabla} \psi|^{2}+\frac{1}{2}|X|^{2}|\psi|^{2}+\frac{3}{2} \kappa^{2}|\psi|^{2}+\frac{1}{2} X\left(|\psi|^{2}\right)\right] .
$$

We show the integrand of $\mathfrak{R}$ is pointwise positive. When $X=0$ at $p$, this is certainly true. So we may assume $X \neq 0$, and thus

$$
|\widehat{\nabla} \psi|^{2} \geq \frac{1}{|X|^{2}}\left|\bar{\nabla}_{X} \psi+\frac{\sqrt{-1}}{2} \kappa c(X) \psi\right|^{2} \geq \frac{1}{|X|^{2}}\left(\left|\bar{\nabla}_{X} \psi\right|-\frac{1}{2} \kappa|X||\psi|\right)^{2} .
$$

Also,

$$
X\left(|\psi|^{2}\right)=\left\langle\bar{\nabla}_{X} \psi, \psi\right\rangle+\left\langle\psi, \bar{\nabla}_{X} \psi\right\rangle \geq-2\left|\bar{\nabla}_{X} \psi \| \psi\right|
$$

So the integrand of $\mathfrak{R}$ is not less than

$$
\frac{2}{3} \frac{1}{|X|^{2}}\left|\bar{\nabla}_{X} \psi\right|^{2}-\frac{2}{3} \frac{1}{|X|}\left|\bar{\nabla}_{X} \psi\right| \kappa|\psi|+\frac{5}{3} \kappa^{2}|\psi|^{2}+\frac{1}{2}|X|^{2}|\psi|^{2}-\left|\bar{\nabla}_{X} \psi\right||\psi|,
$$

which can be completed to a sum of squares

$$
\frac{1}{6}\left(\frac{1}{|X|}\left|\bar{\nabla}_{X} \psi\right|-2 \kappa|\psi|\right)^{2}+\frac{1}{2}\left(\frac{1}{|X|}\left|\bar{\nabla}_{X} \psi\right|-|X||\psi|\right)^{2}+\kappa^{2}|\psi|^{2} .
$$

Therefore,

$$
\int_{\tilde{M}_{r}}|\widehat{D} \psi|^{2} \geq \frac{1}{3} \int_{\tilde{M}_{r}}|\widehat{\nabla} \psi|^{2}
$$

On the other hand,

$$
\int_{\tilde{M}_{r}}|\widehat{\nabla} \psi|^{2}=\int_{\tilde{M}_{r}}|\bar{\nabla} \psi|^{2}+\frac{3}{4} \kappa^{2} \int_{\tilde{M}_{r}}|\psi|^{2}
$$

Therefore, $B$ is coercive on $W^{1,2}$.

Since $B$ is bounded and coercive on $W^{1,2}$, by Lax-Milgram, there exists a $\phi_{1} \in W^{1,2}$ such that for all $\psi \in W^{1,2}$ (6.3) holds. Thus $\phi_{a}=\phi_{1}+\phi_{a, 0}$ satisfies $\int_{\tilde{M}}\left\langle\widehat{D} \psi, \widehat{D} \phi_{a}\right\rangle=0$ for all $\psi \in W^{1,2}$. 
Set $\Phi=\widehat{D} \phi_{a}$. As in the previous case, integration by parts implies

$$
\widehat{D}^{*} \Phi=\bar{D} \Phi+\frac{3}{2} \sqrt{-1} \kappa \Phi=0
$$

weakly. The rest of the proof is similar to the previous case.

\subsection{Proofs of Theorem 1.3 and 1.4}

The positivity of the total mass $\lim _{r \rightarrow \infty} m_{r}\left(\phi_{a}\right)$ can be restated as

Corollary 6.3. Under the assumption of Theorem 6.1 or 6.2

$$
\lim _{r \rightarrow \infty} \int_{\Sigma_{r}}\left(H_{0}-\mathcal{H}\right) \mathbf{X}
$$

is a future-directed time-like vector, where $\boldsymbol{X}$ is the position vector of $\Sigma_{r}$ as in Proposition 4.1.

Proof. By Theorem 6.1 or 6.2, there exists a Killing-harmonic spinor $\phi_{a}$ on $\tilde{M}$ that is asymptotic to $\phi_{a, 0}=A \phi_{a, 0}^{\prime}$ in the sense of (6.1). For the Killingharmonic spinor $\phi_{a}$, by Proposition 3.1

$$
\int_{\Sigma_{r}}\left\langle\phi_{a},\left(\widehat{\nabla}_{\nu_{r}}+c^{\prime \prime}\left(\nu_{r}\right) \cdot \widehat{D}\right) \phi_{a}\right\rangle_{g} \geq 0
$$

and thus we have

$$
\lim _{r \rightarrow \infty} \int_{\Sigma_{r}}\left\langle\phi_{a, 0},\left(\widehat{\nabla}_{\nu_{r}}+c^{\prime \prime}\left(\nu_{r}\right) \cdot \widehat{D}\right) \phi_{a, 0}\right\rangle_{g} \geq 0
$$

By Proposition 3.1 and Corollary 3.5, this expression for $\phi_{a, 0}$ is the same as

$$
\lim _{r \rightarrow \infty} \int_{\Sigma_{r}}\left(H_{0}-\mathcal{H}\right)\left|\phi_{a, 0}\right|^{2}=\lim _{r \rightarrow \infty} \int_{\Sigma_{r}}\left(H_{0}-\mathcal{H}\right)\left|\phi_{a, 0}^{\prime}\right|^{2}
$$

which, by Proposition 4.1, implies

$$
-\kappa \lim _{r \rightarrow \infty} \int_{\Sigma_{r}}\left(H_{0}-\mathcal{H}\right) \mathbf{X} \cdot \zeta(a) \geq 0
$$

for any $a$. Since $\zeta$ maps onto the light cone, this implies that the Lorentz product of $\lim _{r \rightarrow \infty} \int_{\Sigma_{r}}\left(H_{0}-\mathcal{H}\right) \mathbf{X}$ with any future-directed light-like vector is non-positive. 
We are ready to prove Theorem 1.3 and 1.4.

Proof. In either case, we construct the manifold $\left(M, g^{\prime \prime}\right)$ with the appropriate $\mathcal{H}(0)$ according to Theorem 6.1 or 6.2 , we solve the Equation (5.3) on $\left(M, g^{\prime \prime}\right)$, and we obtain a vector-valued function $\mathbf{W}$.

Theorem 6.1 and 6.2 also imply, by Proposition 5.3 and Lemma 5.4, that

$$
\lim _{r \rightarrow \infty} \int_{\Sigma_{r}}\left(H_{0}-\mathcal{H}\right) \mathbf{W} \cdot \zeta(a) \geq 0 .
$$

Now let $\mathbf{W}^{0}$ be the solution of $\mathbf{W}$ at $r=0$, i.e., $\mathbf{W}(0)$. By the monotonicity formula (Proposition 5.3),

$$
\int_{\Sigma_{0}}\left(H_{0}-\mathcal{H}\right) \mathbf{W}^{0} \cdot \zeta(a) \geq \lim _{r \rightarrow \infty} \int_{\Sigma_{r}}\left(H_{0}-\mathcal{H}\right) \mathbf{W} \cdot \zeta(a) \geq 0,
$$

and the theorems are proved.

\section{Acknowledgement}

The first author is supported by NSF grants DMS-0306049 and DMS-0605115 and a Sloan research fellowship. The second author is supported by NSF grants DMS-0354737, DMS-0306600, and DMS-0628341.

\section{References}

[1] L. Andersson and M. Dahl, Scalar curvature rigidity for asymptotically locally hyperbolic manifolds. Ann. Global Anal. Geom. 16 (1998), 1-27.

[2] R. Bartnik, Quasi-spherical metrics and prescribed scalar curvature. J. Differential Geom. 37 (1993), 31-71.

[3] H. Baum, Complete Riemannian manifolds with imaginary Killing spinors. Ann. Global Anal. Geom. 7(3) (1989), 205-226.

[4] J. D. Brown, J. W. York Jr., Quasilocal energy in general relativity. Mathematical aspects of classical field theory (Seattle, WA, 1991), 129-142, Contemp. Math., 132, Amer. Math. Soc., Providence, RI, 1992.

[5] J. D. Brown and J. W. York Jr., Quasilocal energy and conserved charges derived from the gravitational action. Phys. Rev. D (3) 47(4) (1993), 1407-1419. 
[6] R. L. Bryant, Surfaces of mean curvature one in hyperbolic space. Théorie des variéés minimales et applications (Palaiseau, 1983-1984). Astérisque No. 154-155 (1987), 12, 321-347, 353 (1988).

[7] P. T. Chruściel and M. Herzlich, The mass of asymptotically hyperbolic Riemannian manifolds. Pacific J. Math. 212(2) (2003), 231-264.

[8] P. T. Chruściel and G. Nagy, The mass of spacelike hypersurfaces in asymptotically anti-de Sitter space-times. Adv. Theor. Math. Phys. 5 (2002), 697-754.

[9] G. Huisken and S.-T. Yau, Definition of center of mass for isolated physical systems and unique foliations by stable spheres of constant mean curvature. Invent. Math. 124 (1996), 281-311.

[10] J. Kijowski, A simple derivation of canonical structure and quasi-local Hamiltonians in general relativity. Gen. Relativity Gravitation 29(3) (1997), 307-343.

[11] C.-C. M. Liu and S.-T. Yau, Positivity of quasi-local mass. Phys. Rev. Lett. 90(23) (2003), 231102, 4.

[12] C.-C. M. Liu and S.-T. Yau, Positivity of quasi-local mass II. J. Amer. Math. Soc. 19(1) (2006), 181-204.

[13] P. Miao, Positive mass theorem on manifolds admitting corners along a hypersurface. Adv. Theor. Math. Phys., 6(6) (2002), 1163-1182.

[14] M. Min-Oo, Scalar curvature rigidity of asymptotically hyperbolic spin manifolds. Math. Ann. 285 (1989), 527-539.

[15] N. Ó. Murchadha, L. B. Szabados and K. P. Tod, Phys. Rev. Lett 92, (2004), 259001.

[16] T. Parker and C. H. Taubes, On Witten's proof of the positive energy theorem. Commun. Math. Phys. 84(2) (1982), 223-238.

[17] A. V. Pogorelov, Some results on surface theory in the large. Adv. Math. $\mathbf{1}$ (fasc. 2) (1964), 191-264.

[18] Y. Shi and L.-F. Tam, Positive mass theorem and the boundary behavior of compact manifolds with nonnegative scalar curvature. J. Differential Geom. 62(1) (2002), 79-125.

[19] R. Schoen and S.-T. Yau, Positivity of the total mass of a general spacetime. Phys. Rev. Lett. 43(20) (1979), 1457-1459. 
[20] R. Schoen and S.-T. Yau, On the proof of the positive mass conjecture in general relativity. Commun. Math. Phys. 65(1) (1979), 45-76.

[21] R. Schoen and S. T. Yau, Proof of the positive mass theorem. II. Comm. Math. Phys. 79(2) (1981), 231-260.

[22] R. Schoen and S.-T. Yau, Proof that the Bondi mass is positive. Phys. Rev. Lett. 48(6) (1982), 369-371.

[23] B. Smith and G. Weinstein, On the connectedness of the space of initial data for the Einstein equations. Electron. Res. Announc. Amer. Math. Soc. 6 (2000), 52-63.

[24] X. Wang, The mass of asymptotically hyperbolic manifolds. J. Differential Geom. 57 (2001), 273-299.

[25] E. Witten, A new proof of the positive energy theorem. Commun. Math. Phys. 80(3) (1981), 381-402.

[26] X. Zhang, A definition of total energy-momenta and the positive mass theorem on asymptotically hyperbolic 3-manifolds I. Comm. Math. Phys $\mathbf{2 4 9}(3)$ (2004), 529-548.

Department of Mathematics,

Columbia University,

2990 BROADWAY,

NEW YORK,

NY10027, USA

E-mail address: mtwang@cpw.math.columbia.edu

Department of Mathematics,

HARVARD UNIVERSITY,

1 OXFord StreEt,

CAmbridge,

MA 02138, USA

E-mail address: yau@math.harvard.edu

Received February 20, 2006 